\title{
Viscosity solutions for a model of contact line motion
}

\author{
KARL GLASNER \\ Department of Mathematics, The University of Arizona, \\ 617 N. Santa Rita, Tucson, AZ 85721, USA \\ E-mail:kglasner@math.arizona.edu \\ INWON C. KIM \\ Department of Mathematics, UCLA, Los Angeles, CA 90095, USA \\ E-mail:ikim@math.ucla.edu
}

[Received 21 August 2007 and in revised form 25 April 2008]

\begin{abstract}
This paper considers a free boundary problem that describes the motion of contact lines of a liquid droplet on a flat surface. The elliptic nature of the equation for droplet shape and the monotonic dependence of contact line velocity on contact angle allows us to introduce a notion of "viscosity" solutions for this problem. Unlike similar free boundary problems, a comparison principle is only available for a modified short-time approximation because of the constraint that conserves volume. We use this modified problem to construct viscosity solutions to the original problem under a weak geometric restriction on the free boundary shape. We also prove uniqueness provided there is an upper bound on front velocity.
\end{abstract}

\section{Introduction}

This paper is concerned with solutions of the free boundary problem in $\mathbb{R}^{N} \times[0, \infty)$,

$$
\left\{\begin{array}{l}
-\Delta u(\cdot, t)=\lambda(t ; u) \quad \text { in }\{u(\cdot, t)>0\}, \\
\int_{\{u(\cdot, t)>0\}} u(x, t) \mathrm{d} x=V_{0}, \\
V=F(|D u|) \quad \text { on } \partial\{u>0\}, \\
u(\cdot, 0)=u_{0},
\end{array}\right.
$$

where $F: \mathbb{R}^{+} \rightarrow \mathbb{R}$ is continuous and strictly increasing. Here $V=V(x, t)$ denotes the outward normal velocity of the free boundary $\partial\{u>0\}$ of $u$ at $(x, t)$. In spatial dimension $N=2$, this problem describes the motion of a liquid droplet on a planar surface whose free surface height is $u(x, t)$ and volume is $V_{0}[\mathrm{Gr}, \mathrm{Ho}, \mathrm{G} 1]$. In this context the positive phase $\{u>0\}$ denotes the wet region and the free boundary denotes the contact line between the drop and the surface. The first equation in $(P)$ defines the shape of a quasi-static droplet. The second equation is a volume conservation condition which is enforced by a suitable choice of the Lagrange multiplier $\lambda(t ; u)$ (which is physically the hydrostatic pressure). The third equation in $(P)$ defines the contact line motion by a relationship between the free boundary normal velocity $V=u_{t} /|D u|$ and the "apparent" contact angle $|D u|$. 
The initial condition for the evolution is specified by an open, bounded set $\Omega_{0} \subset \mathbb{R}^{N}$ and volume $V_{0}$. The initial droplet shape $u_{0}: \mathbb{R}^{N} \rightarrow \mathbb{R}^{+}$is the smallest (weak) solution of

$$
\left\{\begin{array}{l}
-\Delta u_{0}=\lambda_{0}>0 \quad \text { in } \Omega_{0}, \\
u_{0}=0 \text { in } \mathbb{R}^{N}-\bar{\Omega}_{0},
\end{array}\right.
$$

where by linearity $\lambda_{0}>0$ can be chosen to satisfy any volume constraint. Also, given $\lambda_{0}>0, u_{0}$ is uniquely determined by

$$
u_{0}:=\inf \left\{v:-\Delta v \geqslant \lambda_{0} \text { in } \Omega_{0}, v \geqslant 0 \text { in } \mathbb{R}^{N}\right\} .
$$

Many formulas for the constitutive velocity relation $F$ appear in the literature (e.g. [T]). The present paper focuses on the most widely used one $[\mathrm{V}, \mathrm{C}]$ ),

$$
F(|D u|):=|D u|^{3}-1 .
$$

The techniques which we use for global existence of solutions, however, only rely on the fact that $F$ is continuous and strictly increasing.

The free boundary problem $(P)$ has been used as a fundamental model for contact line motion for the last 30 years. Mathematical understanding of this problem has been slowly accruing in the form of numerical methods [G1, Hu], stability calculations [Ho] and homogenized dynamics [G2]. On the other hand, very little is known for $(P)$ in terms of rigorous analysis. To the best of our knowledge the short-time existence of classical solutions has not been established. Furthermore, no notion of weak or generalized solutions has yet been put forth. There are, however, compelling reasons to consider non-classical solutions to this free boundary problem. Numerical (and even physical) experiments indicate that the free boundary evolution with initially convex positive phase develops corners (see Figure 11. Of course, other more standard topological singularities of the positive phase, such as splitting and reconnection, are possible as well (in fact we demonstrate this must happen for certain initial data, see Lemma B.6). Our results address only the former type of singularity. There is a good reason for this: during splitting of the free boundary, for example, the model itself breaks down since separate volume constraints for each connected component would be required. While there may be a more general model that admits changes in topology, we do not address this here.

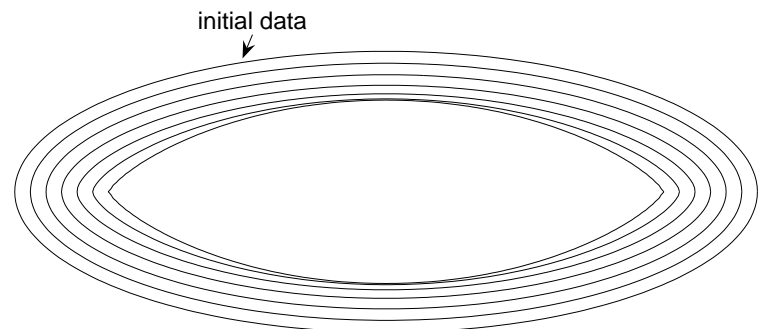

FIG. 1. Development of a nonsmooth corner in the free boundary, using the numerical method in [G1].

Originally invented by Crandall and Lions [CL] for Hamilton-Jacobi equations, viscosity solutions allow for singularities of their level sets, and enjoy strong stability properties under various limits. The notion of viscosity solutions has been applied to a variety of free boundary problems that 
satisfy a comparison principle, which states that if one solution is smaller than the other at one time, then the order is preserved for later times (see, e.g., [K2]). For example, in [K1] a notion of viscosity solutions was introduced for Hele-Shaw and Stefan problems with zero surface tension.

In this paper we define a notion of "viscosity" solutions for problem $(P)$ (see Section 3), and we show that it is well-posed in the sense of existence and uniqueness of solutions. Furthermore, under a moderate geometric restriction (see condition $(I)$ in Section 3.3 and Appendix B), solutions will exist for all time. Below is the summary of the main result (see Theorem 3.8 and Corollary 6.4):

THEOREM 1.1 Suppose $\Omega_{0}$ is star-shaped with respect to a ball $B$ it contains. Then the following holds:

(a) A solution of $(P)$ exists in $\mathbb{R}^{n} \times[0, T]$ for some maximal time $T>0$.

(b) At the maximal time $T$, the ball $B$ is not entirely in the support of $u$.

(c) If the velocity function $F$ is bounded, the solution is unique, at least until the time when $B$ is not entirely in the support of $u$.

In our case, solutions of $(P)$ do not satisfy a comparison principle directly since the Lagrange multiplier $\lambda(t ; u)$ is time-dependent, thus a straightforward definition of viscosity solutions is more difficult. In particular, the comparison principle we employ only holds for discrete time intervals of an approximating problem $(P)_{n}^{M}$ (see Section 3) which relaxes the constraint and fixes $\lambda$ over small time intervals.

The paper is organized as follows. In Section 2 we define viscosity solutions for problem $(P)$ and a modified problem $(P)^{M}$ which puts an upper bound on the free boundary velocity. We also outline the strategy for constructing solutions of $(P)$ by approximating problems $(P)_{n}^{M}$. In Section 3 the small-time-approximation problem $(\tilde{P})$ is defined, and a comparison principle and existence theorem for this problem are given. We also introduce a geometric restriction $(I)$ and discuss settings for which it is satisfied to yield global-in-time existence. In Section 4 we use the results of Section 3 to show existence of a weak solution for $(P)_{n}^{M}$, and derive regularity properties for $u_{n}^{M}$. In Section 5 we use the equicontinuity of the approximating sequence $\left\{u_{n}^{M}\right\}$ to show that it converges to a viscosity solution as $n, M \rightarrow \infty$. In Section 6 we prove that $u^{M}$ can be obtained as the local uniform limit of the whole sequence $\left\{u_{n}^{M}\right\}$ as $n \rightarrow \infty$, and the solution $u^{M}$ of $(P)^{M}$ is unique. In Appendix A we prove the comparison principle and the existence result for solutions of $(\tilde{P})$ stated in Section 3. Finally, in Appendix B we show that the geometric restriction $(I)$ holds for all times when the initial data is (a) symmetric with respect to two axes or (b) symmetric with respect to one axis and convex in two dimensions.

\section{Definitions and preliminaries}

Consider a domain $D \subset \mathbb{R}^{N}$ and a time interval $I \subset \mathbb{R}^{+}$. For a nonnegative real-valued function $u(x, t)$ defined for $(x, t) \in D \times I$, we will use the notation

$$
\begin{aligned}
\Omega(u) & =\{(x, t) \in D \times I: u(x, t)>0\}, & \Omega_{t}(u) & =\{x \in D: u(x, t)>0\}, \\
\Gamma(u) & =\partial \Omega(u)-\partial(D \times I), & \Gamma_{t}(u) & =\partial \Omega_{t}(u)-\partial D .
\end{aligned}
$$

We call $\Omega(u)$ and $\Gamma(u)$ respectively the positive phase and the free boundary of $u$.

For $x \in \mathbb{R}^{N}$ we also denote by $B_{r}(x)$ the ball of radius $r$ with center $x$ in $\mathbb{R}^{N}$. 


\subsection{Viscosity solutions}

We first define the notion of viscosity solutions for problem $(P)$, with open, bounded initial positive phase $\Omega_{0}$ and initial shape given by (1).

Definition 2.1 A nonnegative function $u(x, t)$ in $Q:=\mathbb{R}^{N} \times[0, \infty)$ is a viscosity solution of $(P)$ in $Q$ with initial positive phase $\Omega_{0}$ and volume $V_{0}$ if the following is true:

1. $u$ is continuous with $u(\cdot, 0)=u_{0}(x)$.

2. At each $t>0,-\Delta u=\lambda(t ; u)$ in $\Omega_{t}(u)$ where $\lambda(t):=\lambda(t ; u)$ is chosen such that

$$
\int u(x, t) \mathrm{d} x=\int u_{0}(x) \mathrm{d} x=V_{0} .
$$

3. For every $\phi \in C^{2,1}(Q)$ such that $u-\phi$ has a local maximum in $\overline{\Omega(u)} \cap\left\{t \leqslant t_{0}\right\}$ at $\left(x_{0}, t_{0}\right) \in \Gamma(u)$ with $|D \phi|\left(x_{0}, t_{0}\right) \neq 0$,

$$
\left(\phi_{t}-|D \phi|\left(|D \phi|^{3}-1\right)\right)\left(x_{0}, t_{0}\right) \leqslant 0 .
$$

4. For every $\phi \in C^{2,1}(Q)$ such that $u-\phi$ has a local minimum in $\overline{\Omega(u)} \cap\left\{t \leqslant t_{0}\right\}$ at $\left(x_{0}, t_{0}\right) \in \Gamma(u)$ with $|D \phi|\left(x_{0}, t_{0}\right) \neq 0$,

$$
\left(\phi_{t}-|D \phi|\left(|D \phi|^{3}-1\right)\right)\left(x_{0}, t_{0}\right) \geqslant 0 .
$$

Note that classical solutions of $(P)$ are also viscosity solutions.

One can similarly define viscosity solutions of a problem which has an imposed upper bound on velocity,

$$
\left\{\begin{array}{l}
-\Delta u(\cdot, t)=\lambda(t ; u) \quad \text { in }\{u>0\}, \\
\int_{\{u>0\}} u \mathrm{~d} x=V_{0}, \\
V=\frac{u_{t}}{|D u|}=\min \left(|D u|^{3}-1, M\right) \quad \text { on } \partial\{u>0\} .
\end{array}\right.
$$

Since the upper bound is arbitrary, there is no loss of generality in the physical problem where one expects finite speeds. This modification considerably simplifies our analysis of proving uniqueness in Section 6.

Note that $(P)$ does not satisfy a comparison principle: since $\Omega_{s} \subset \Omega_{t}$ implies $\lambda(t) \leqslant \lambda(s)$, one cannot use the maximum principle to conclude that $u(x, t) \leqslant u(x, s)$. Therefore the usual viscosity solution approach must be modified. To do this, we consider the "discrete time approximation" problem

$$
\begin{cases}-\Delta u_{n}^{M}(\cdot, t)=\lambda_{n, M}\left(k t_{n}\right) & \text { in }\left\{u_{n}^{M}>0\right\} \cap\left[k t_{n},(k+1) t_{n}\right), \\ V=\min \left(F\left(\left|D u_{n}^{M}\right|\right), M\right) & \text { on } \partial\left\{u_{n}^{M}>0\right\} \\ u^{M}(x, 0)=u_{0}(x), & \end{cases}
$$

where $t_{n}:=2^{-n}$ and $\lambda_{n, M}\left(k t_{n}\right)$ is chosen so that

$$
\int u_{n}^{M}\left(\cdot, k t_{n}\right) \mathrm{d} x=V_{0} \quad \text { for } k=0,1,2, \ldots
$$

This problem will satisfy the desired comparison principle in each time interval $\left[k t_{n},(k+1) t_{n}\right)$. Note that if $\Gamma_{t}\left(u_{n}\right)$ and $u_{n}^{M}$ change continuously in time, then by (2.1),

$$
u_{n}^{M}\left(x, k t_{n}\right)=\frac{\lambda_{n, M}\left(k t_{n}\right)}{\lambda_{n, M}\left((k-1) t_{n}\right)} \lim _{t \uparrow k t_{n}} u_{n}^{M}(x, t) .
$$


To construct a viscosity solution of $(P)$, we first construct the solution $u_{n}^{M}$ of $(P)_{n}^{M}$ by finding a viscosity solution in $\left[k t_{n},(k+1) t_{n}\right)$ on each interval and restart at $t=(k+1) t_{n}$ using $(2.2)$. We will then show that $u_{n}^{M}$ and $\Omega_{t}\left(u_{n}^{M}\right)$ converge uniformly as $n, M$ go to infinity to a viscosity solution of the original problem.

\section{The small-time problem and a comparison principle}

As a small-time approximation of $(P)$, we consider

$$
\left\{\begin{array}{l}
-\Delta u(\cdot, t)=\lambda \quad \text { in }\{u>0\}, \\
V=\frac{u_{t}}{|D u|}=F(|D u|) \quad \text { on } \partial\{u>0\},
\end{array}\right.
$$

where $\lambda$ is a prescribed constant, rather than determined by an additional constraint. For purposes of this section only, we allow $F:[0, \infty) \rightarrow \mathbb{R}$ to be any continuous, increasing function. In particular, if $F$ is replaced with $\min (F, M)$, then a solution $u_{n}^{M}$ of $(P)_{n}^{M}$ will also solve $(\tilde{P})$ with $\lambda=\lambda\left(k t_{n}\right)$ on intervals $\left[k t_{n},(k+1) t_{n}\right)$.

Let $Q=\mathbb{R}^{N} \times(0, \infty)$.

DEFINITION 3.1 A nonnegative upper semicontinuous function $u$ defined in $Q$ is a viscosity subsolution of $(\tilde{P})$ if

(a) for each $a<T<b$ the set $\overline{\Omega(u)} \cap\{t \leqslant T\}$ is bounded;

(b) for every $\phi \in C^{2,1}(Q)$ such that $u-\phi$ has a local maximum in $\overline{\Omega(u)} \cap\left\{t \leqslant t_{0}\right\} \cap Q$ at $\left(x_{0}, t_{0}\right)$,

(i) $-\Delta \phi\left(x_{0}, t_{0}\right) \leqslant \lambda$ when $u\left(x_{0}, t_{0}\right)>0$,

(ii) $\left(\phi_{t}-|D \phi| F(|D \phi|)\right)\left(x_{0}, t_{0}\right) \leqslant 0$ if $\left(x_{0}, t_{0}\right) \in \Gamma(u)$ when $-\Delta \phi\left(x_{0}, t_{0}\right)>\lambda$.

Note that because $u$ is only upper semicontinuous there may be points of $\Gamma(u)$ at which $u$ is positive.

DEFINITION 3.2 A nonnegative lower semicontinuous function $v$ defined in $Q$ is a viscosity supersolution of $(\tilde{P})$ if for every $\phi \in C^{2,1}(Q)$ such that $v-\phi$ has a local minimum in $Q \cap\left\{t \leqslant t_{0}\right\}$ at $\left(x_{0}, t_{0}\right)$,

(i) $-\Delta \phi\left(x_{0}, t_{0}\right) \geqslant \lambda$ if $v\left(x_{0}, t_{0}\right)>0$,

(ii) if $\left(x_{0}, t_{0}\right) \in \Gamma(v),|D \phi|\left(x_{0}, t_{0}\right) \neq 0$ and $-\Delta \phi\left(x_{0}, t_{0}\right)<\lambda$, then $\left(\phi_{t}-|D \phi| F(|D \phi|)\right)\left(x_{0}, t_{0}\right)$ $\geqslant 0$.

For a nonnegative real-valued function $f(x, t)$ in a cylindrical domain $D \times(a, b)$ we define

$$
f^{*}(x, t):=\limsup _{(\xi, s) \in D \times(a, b) \rightarrow(x, t)} f(\xi, s), \quad f_{*}(x, t):=\liminf _{(\xi, s) \in D \times(a, b) \rightarrow(x, t)} f(\xi, s) .
$$

DEFINITION 3.3 A lower semicontinuous function $u$ is a viscosity solution of $(\tilde{P})$ if $u$ is a viscosity supersolution and $u^{*}$ is a viscosity subsolution of $(\tilde{P})$. Moreover, $u$ is a viscosity solution of $(\tilde{P})$ with initial positive phase $\Omega_{0}$ if $\Omega_{0}\left(u^{*}\right)=\Omega_{0}(u)=\Omega_{0}$.

For later use we show that free boundaries of solutions of $(\tilde{P})$ do not "jump" at any positive time.

Lemma 3.4 Let $v$ solve $(\tilde{P})$ in $Q$. Then for $x_{0} \in \Gamma_{t_{0}}(v)$ with $t_{0}>0$, there exist $x_{n} \in \Gamma_{t_{n}}(v)$ with $t_{n}<t_{0}$ such that $x_{n} \rightarrow x_{0}$ and $t_{n} \rightarrow t_{0}$ as $n \rightarrow \infty$. 
Proof. Suppose otherwise. Then there exists $r>0$ and a sequence of $t_{k}$ converging to $t_{0}$ such that for large $k$, either (i) $v\left(\cdot, t_{k}\right)=0$ in $B_{r}\left(x_{0}\right)$ or (ii) $B_{r}\left(x_{0}\right) \subset \Omega_{t_{k}}(v)$.

First note that, due to Definition 3.1(a), $M=\sup _{\mathbb{R}^{n} \times[0, \infty)} v$ is finite. If (i) holds, we construct a barrier function $\phi(x, t)$ in $\Sigma:=B_{r+2}\left(x_{0}\right) \times\left[t_{k}, t_{0}\right]$ such that

$$
\begin{cases}-\Delta \phi(\cdot, t)=2 \lambda \quad \text { in } B_{r+2}\left(x_{0}\right)-B_{r(t)}\left(x_{0}\right), & \text { on } \partial B_{r(t)}\left(x_{0}\right), \\ \phi(\cdot, t)=0 & \text { on } \partial B_{r+2}\left(x_{0}\right),\end{cases}
$$

where $r(t)=r+\left(t_{0}-t\right) /\left(2\left(t_{0}-t_{k}\right)\right)$. (Note that $r(t)$ is positive for $t_{k} \leqslant t \leqslant t_{0}$.) Since $r(t) \in$ $[r, r+1]$ for $t \in\left[t_{k}, t_{0}\right]$, we have $|D \phi| \leqslant C_{0}=C_{0}(M, r)$ on $\partial B_{r(t)}\left(x_{0}\right)$, and thus

$$
\phi_{t}-|D \phi| F(|D \phi|) \geqslant\left(2\left(t_{0}-t_{k}\right)\right)^{-1}-C_{0} F\left(C_{0}\right) \quad \text { on } \partial B_{r(t)}\left(x_{0}\right) \text {. }
$$

Hence if $t_{k}$ is sufficiently close to $t_{0}$, then $\phi$ is a supersolution of $(\tilde{P})$ in $\Sigma$ with $\phi \in C^{2,1}(\bar{\Omega}(\phi))$. Using Definition 3.1, one can check that $v \leqslant \phi$ in $\Sigma$ and in particular $x_{0}$ lies in the interior of the zero set of $v\left(\cdot, t_{0}\right)$, a contradiction.

If (ii) holds, we construct the barrier $\varphi(x, t)$ in $\Sigma$ such that

$$
-\Delta \varphi(\cdot, t)=\lambda \quad \text { in } B_{r(t)}\left(x_{0}\right), \quad \varphi(\cdot, t)=0 \quad \text { in } \mathbb{R}^{N}-B_{r(t)}\left(x_{0}\right)
$$

where $r(t)$ is given above. If $t_{k}$ is sufficiently close to $t_{0}$, then $\varphi$ is a subsolution of $(\tilde{P})$ in $\Sigma$ with $\varphi \in C^{2,1}(\bar{\Omega}(\varphi))$ and with smooth positive phase. Hence using Definition 3.2, one can check that $v \geqslant \varphi$ in $\Sigma$ and in particular $x_{0}$ lies in the interior of $\Omega_{t_{0}}(v)$, a contradiction.

\subsection{Convolutions}

An important set of tools for the subsequent analysis are the inf- and sup-convolutions over space balls. These are employed to obtain larger or smaller sub- and supersolutions from existing sub- and supersolutions.

LEMMA 3.5 (a) If $u$ is a viscosity subsolution of $(\tilde{P})$, then the sup-convolution

$$
\tilde{u}(x, t):=\sup _{y \in B_{r-c t}(x)} u(y, t)
$$

is a viscosity subsolution of $(\tilde{P})$ with $F(|D u|)$ replaced by $F(|D u|)-c$, as long as $r-c t>0$. (b) If $u$ is a supersolution of $(\tilde{P})$ then the inf-convolution

$$
\tilde{u}(x, t):=\inf _{y \in B_{r-c t}(x)} u(y, t)
$$

is a viscosity supersolution of $(\tilde{P})$ with $F(|D u|)$ replaced by $F(|D u|)+c$, as long as $r-c t>0$.

Proof. We only prove (a).

First suppose $\tilde{u}(\cdot, t)-\phi(\cdot, t)$ has a local maximum at $x_{0} \in \Omega_{t}(\tilde{u})$. By the definition of $\tilde{u}$, $u(\cdot, t)-\phi\left(\cdot+\left(x_{0}-y_{0}\right), t\right)$ has a local maximum at $y_{0}$, where $y_{0} \in B_{r-c t}\left(x_{0}\right)$ and $\tilde{u}\left(x_{0}, t\right)=u\left(y_{0}, t\right)$. Since $u$ is a viscosity solution of $(\tilde{P})$, it follows that $-\Delta \phi\left(x_{0}, t\right) \leqslant \lambda$. Hence our claim is proved. 
Next suppose that $\tilde{u}(\cdot, t)-\phi(\cdot, t)$ has a local maximum zero in $\bar{\Omega}(\tilde{u}) \cap\left\{t \leqslant t_{0}\right\}$ at $\left(x_{0}, t_{0}\right) \in \Gamma(\tilde{u})$ with $|D \phi|\left(x_{0}, t_{0}\right) \neq 0$. By definition of $\tilde{u}$, the function $u-\tilde{\phi}$, where

$$
\tilde{\phi}(x, t):=\phi\left(x+\left(1-c\left|x_{0}-y_{0}\right|^{-1}\left(t-t_{0}\right)\right)\left(x_{0}-y_{0}\right), t\right),
$$

has a local maximum in $\bar{\Omega}(u) \cap\left\{t \leqslant t_{0}\right\}$ at $\left(y_{0}, t_{0}\right) \in \Gamma(u)$, where $\left|y_{0}-x_{0}\right|=r-c t_{0}$ and $\tilde{u}\left(x_{0}, t\right)=u\left(y_{0}, t\right)$. Note that $B_{r-c t_{0}}\left(y_{0}\right)$ lies in $\Omega_{t_{0}}(\tilde{u})$ and touches $\Gamma_{t_{0}}(\tilde{u})$ at $x_{0}$. Since $\tilde{u}$ touches $\phi$ from below, it follows that $y_{0}-x_{0}$ is parallel to the direction of $D \phi\left(x_{0}, t_{0}\right)$. Since $u$ is a viscosity solution of $(\tilde{P})$, it follows that

$$
\frac{\tilde{\phi}_{t}}{|D \tilde{\phi}|}\left(y_{0}, t_{0}\right)=\frac{\phi_{t}}{|D \phi|}\left(x_{0}, t_{0}\right)+c \leqslant F(|D \tilde{\phi}|)\left(y_{0}, t_{0}\right)=F(|D \phi|)\left(x_{0}, t_{0}\right) .
$$

\subsection{Comparison principle}

Here we state the comparison principle for viscosity solutions of $(\tilde{P})$.

Definition 3.6 We say that a pair of functions $u_{0}, v_{0}: \bar{D} \rightarrow[0, \infty)$ are (strictly) separated (denoted by $u_{0} \prec v_{0}$ ) in $D$ if

(i) the support of $u_{0}, \operatorname{supp}\left(u_{0}\right)=\overline{\left\{u_{0}>0\right\}}$, restricted to $\bar{D}$ is compact,

(ii) the functions are strictly ordered in the support of $u_{0}$ :

$$
u_{0}(x)<v_{0}(x) \quad \text { in }\left\{u_{0} \geqslant 0\right\} .
$$

Variations of the following theorem, whose proof is deferred to Appendix A, will be used later in the paper.

THEOREM 3.7 (Comparison principle) Let $u, v$ be respectively viscosity sub- and supersolutions of $(\tilde{P})$ in $\Sigma=D \times(a, b)$ with $u(\cdot, 0) \prec v(\cdot, 0)$ in $D$. If $u(\cdot, t)<v(\cdot, t)$ on $\partial D$ for $a<t<b$, then $u(\cdot, t) \prec v(\cdot, t)$ in $D$ for $t \in[0, T)$.

\subsection{A geometric restriction and global existence}

As discussed in the introduction, one cannot expect viscosity solutions to exist for all time in every circumstance. This fact will be encoded into a restriction on the shape of the positive phase, which is the following: We say that a domain $\Omega \subset \mathbb{R}^{N}$ is star-shaped with respect to a point $p_{0} \in \Omega$ if the line segments connecting $p_{0}$ to boundary points $q \in \partial \Omega$ lie in $\Omega$.

The following theorem, whose proof is deferred to Appendix A, establishes existence for small times of star-shaped solutions to problem $(\tilde{P})$. We will later prove short-time existence for the full problem as well.

THEOREM 3.8 There exists a viscosity solution of $(\tilde{P})$ in $Q$ with initial positive phase $\Omega_{0}$ if $\Omega_{0}$ is star-shaped with respect to $B_{r}(0)$ for some $r>0$.

For long time existence for the full problem $(P)$, we need to ensure that star-shapedness is preserved. Below we prove this is true provided the free boundary does not collapse in on the "center"; that is, there must always be some ball in $\Omega_{t}$ so that $\Omega_{t}$ is star-shaped with respect to points in that ball. In particular, this allows us to side-step issues involved with topological changes, 
such as when the free boundary pinches off. The precise requirement is the following: there exists $r>0$ so that solutions $v(x, t)$ satisfy

$$
\left\{\begin{array}{l}
\Omega_{0}(v) \text { is star-shaped with respect to } B_{r}(0), \\
B_{r}(0) \subset \Omega_{t}(v)
\end{array}\right.
$$

We will in general invoke requirement $(I)$ when referring to approximating solutions $u_{n}^{M}$ (see Definition 4.1), but we could just as well suppose that $(I)$ holds for the limits as $n, M \rightarrow \infty$, that is, for viscosity solutions to the full problem. We briefly detail some natural cases where $(I)$ is expected to hold:

1. If the free boundary is expanding, then $B_{r}(0)$ will always be in $\Omega_{t}\left(u_{n}^{M}\right)$, and therefore the free boundary will always be star-shaped. Conversely, a contracting free boundary would still satisfy (I) with possibly different $r$ up to the point at which $B_{r}(0)$ was entirely outside the positive phase.

2. A convex positive phase is star-shaped with respect to every ball, and therefore remains that way if it is contracting. In other words, convexity is preserved for strictly contracting free boundaries. We also suspect, but cannot prove, that this is the case for expanding free boundaries.

3. If the initial data has certain symmetries, $(I)$ is guaranteed for all times. Details of this are given in Appendix B.

4. Since there is a lower bound on the free boundary velocity, $B_{r}(0)$ will at least stay inside $\Omega_{t}$ for a short time. For short-time existence, we can therefore always assume $(I)$ holds, so long as the initial data is star-shaped.

We will now prove that star-shapedness is preserved as long as $(I)$ holds in problem $(\tilde{P})$, and later observe the same is true for the full problem. Therefore if $(I)$ is preserved by the evolution, we will be able to obtain global existence and uniqueness.

LemMA 3.9 Suppose that $v$ solves $(\tilde{P})$ with $F(|D v|)=\min \left(|D v|^{3}-1, M\right)$ and condition $(I)$ is satisfied. Then $\Omega_{t}(v)$ is star-shaped for all $t>0$.

Proof. Let $x_{0} \in B_{r}(0)$. We claim that for all $x$,

$$
v(x, t) \leqslant(1+\epsilon)^{2} v\left(\frac{x-x_{0}}{1+\epsilon}+x_{0}, t\right) \text { for any } \epsilon>0 .
$$

For $t \in[0, c /(2 M+2)]$ define

$$
\tilde{v}(x, t)=\inf _{y \in B_{c \epsilon-(2 M+2) \epsilon t}(x)}(1+\epsilon)^{2} v\left(\frac{y-x_{0}}{1+\epsilon}+x_{0}, t\right)
$$

where $c$ (which only depends on $r$ ) is chosen small enough so that $v \prec \tilde{v}$ at $t=0$.

Notice this is just an inf-convolution of a rescaled version of $v$, which is easily checked to be a supersolution, so Lemma 3.5 applies. Therefore,

$$
\begin{aligned}
\frac{\tilde{v}_{t}}{|D \tilde{v}|} & =(1+\epsilon) \min \left(|D v|^{3}-1, M\right)+(2 M+2) \epsilon \\
& \geqslant(1+\epsilon) \min \left((1-3 \epsilon)|D \tilde{v}|^{3}-1, M\right)+(2 M+2) \epsilon>\min \left(|D \tilde{v}|^{3}-1, M\right) .
\end{aligned}
$$


Moreover, $-\Delta \tilde{v}(\cdot, t) \geqslant \lambda$ in $\Omega_{t}(\tilde{v})$ due to Lemma 3.5. Hence $\tilde{v}$ is a supersolution of $(\tilde{P})$. Now Theorem 3.7 applies to $v$ and $\tilde{v}$ in $\mathbb{R}^{N} \times[0, c /(2 M+2)]$ to yield $v \leqslant \tilde{v}$ for $0 \leqslant t \leqslant t_{1}:=c /(2 M+2)$, which yields (3.1). Since $\epsilon>0$ in (3.1) is arbitrary, it follows that $v(\cdot, t)$ satisfies (I) if $t \in\left[0, t_{1}\right]$. One can repeat this process indefinitely on time intervals of length $c /(2 M+2)$.

\section{Construction of the approximating sequence}

Our next goal is to construct solutions $u_{n}^{M}$ of the approximating problem $(P)_{n}^{M}$ with star-shaped initial positive phase $\Omega_{0}$ and initial volume $V_{0}$, under condition $(I)$. By definition of $(P)_{n}^{M}, u_{n}^{M}$ is in general discontinuous in time at the endpoints of the time intervals $I_{k}:=\left[k t_{n},(k+1) t_{n}\right)$, and as mentioned before the comparison principle only holds for $u_{n}^{M}$ in small time intervals $I_{k}$ and thus a conventional notion of viscosity solutions will not apply. It is therefore necessary to first establish a weak notion of solutions for $(P)_{n}^{M}$.

DEFINITION $4.1 u_{n}^{M}$ is a weak solution of $(P)_{n}^{M}$ with initial positive phase $\Omega_{0}$ and volume $V_{0}$ for $0 \leqslant t \leqslant(l+1) t_{n}$ where $l \in \mathbb{N}$ if the following holds for $k=0,1, \ldots, l$ :

(i) $u_{n}^{M}(\cdot, 0)=u_{0}$,

(ii) $u_{n}^{M}\left(\cdot, t+k t_{n}\right)$ is a viscosity solution of $(\tilde{P})$ in $\left(0, t_{n}\right]$ with initial positive phase $\Omega_{k t_{n}}\left(u_{n}^{M}\right)$ and $\lambda=\lambda_{n, M}\left(k t_{n}\right)$, where $\lambda_{n, M}\left(k t_{n}\right)$ satisfies (2.1),

(iii) $\Omega_{k t_{n}}\left(u_{n}^{M}\right)$ is continuous from below, that is,

$$
d\left(\Omega_{t}\left(u_{n}^{M}\right), \Omega_{k t_{n}}\left(u_{n}^{M}\right)\right) \rightarrow 0 \quad \text { as } t \uparrow k t_{n} .
$$

Note that due to Lemma $3.9 u_{n}^{M}$ has its positive phase star-shaped in space with respect to $B_{r}(0)$ as long as $B_{r}(0)$ lies in $\Omega_{t}\left(u_{n}^{M}\right)$. It follows that the family of domains $\left\{\Omega_{t}\left(u_{n}^{M}\right)\right\}_{n, t}$ is uniformly Lipschitz in space if they are uniformly bounded (see Remark below Lemma 4.4). Using this fact, in Proposition 4.5 we will show that for weak solutions $u_{n}^{M}$ satisfying $(I), \Omega\left(u_{n}^{M}\right)$ is uniformly Hölder continuous in time. This establishes equicontinuity for the family of functions $\left\{u_{n}^{M}\right\}$ needed to obtain convergence to a solution of $(P)^{M}$ in Section 5 .

First we give an upper bound for $\lambda\left(k t_{n}\right)$ in terms of the circumradius of $\Omega_{k t_{n}}\left(u_{n}^{M}\right)$.

Lemma 4.2 Suppose $u_{n}^{M}$ exists and satisfies (I) with $r>0$ for $0 \leqslant t \leqslant k t_{n}$. Then for $x_{0}$ in $\Gamma_{k t_{n}}\left(u_{n}^{M}\right), \lambda\left(k t_{n}\right) \leqslant C_{0} /\left|x_{0}\right|$, where $C_{0}$ only depends on $r, V_{0}$ and $N$.

Proof. Due to Lemma 3.9 $u_{n}^{M}$ is star-shaped with respect to $B_{r}(0)$. Therefore $\Omega_{k t_{n}}\left(u_{n}^{M}\right)$ contains a cone with vertex $x_{0}$, axis parallel to $x_{0}$ and bottom $B_{r}(0) \cap\left\{x \cdot x_{0}=0\right\}$. It follows that the function $f(x)$ solving

$$
-\Delta f=1 \quad \text { in } \Omega_{k t_{n}}\left(u_{n}^{M}\right), \quad f=0 \quad \text { on } \Gamma_{k t_{n}}\left(u_{n}^{M}\right)
$$

is larger than the superpositions of $h\left(x-k r x_{0} /\left|x_{0}\right|\right), k=1, \ldots,\left|x_{0}\right| / 2 r$, where $h$ solves $-\Delta h=1$ in $B_{r / 2}(0)$ with $h=0$ on $\partial B_{r / 2}(0)$. Thus

$$
\int f(x) \mathrm{d} x \geqslant \frac{\left|x_{0}\right|}{2 r} \int h(x) \mathrm{d} x \geqslant C\left|x_{0}\right| r^{N+2} .
$$

Multiplying by $\lambda\left(k t_{n}\right)$ and noting the definition of $V_{0}$, we can obtain the desired bound.

Lemma 4.3 Suppose $u_{n}^{M}$ satisfies (I), with $r>0$ independent of $n$ and $M$, for $0 \leqslant t \leqslant T$. Define $R(t, n, M):=\sup \left\{|x|: x \in \Omega_{t}\left(u_{n}^{M}\right)\right\}$. Then $R(t, n, M) \leqslant R(T)$ for $t<T$, where $R(T)$ is independent of $n, M$. 
Proof. Let $f$ solve $-\Delta f=2 C_{0}$ in $B_{1}(0)$ with $f=0$ on $\partial B_{1}(0)$, where $C_{0}$ is as given in Lemma 4.2 Let $A$ be the value of $|D f|^{3}$ on $\partial B_{1}(0)$ (note that $f$ is radially symmetric). Next define

$$
h(x, t):=R(t) f\left(\frac{x}{R(t)}\right) \text { with } R(t)=R(0)+A t,
$$

where $R(0)$ is large enough such that $B_{R(0)}(0)$ contains $\bar{\Omega}_{0}$. Note that

$$
-\Delta h=\frac{2 C_{0}}{R(t)} \quad \text { in } B_{R(t)}(0)
$$

with $h=0$ in $\partial B_{R(t)}(0)$ and

$$
\frac{h_{t}}{|D h|}=R^{\prime}(t) \geqslant|D h|^{3}>|D h|^{3}-1 \quad \text { on } \Gamma_{t}(h)
$$

We claim that $\Omega_{t}\left(u_{n}^{M}\right)$ is always strictly contained in $B_{R(t)}(0)$. To see this, suppose otherwise. Then due to the definition of $u_{n}^{M}$ and Lemma 3.4 $\Gamma_{t}\left(u_{n}^{M}\right)$ intersects $\partial B_{R(t)}(0)$ from inside of the ball for the first time at $t=t_{0} \in\left(k t_{n},(k+1) t_{n}\right]$. Choose the smallest ball $B_{R}(0)$ containing $\Omega_{k t_{n}}\left(u_{n}^{M}\right)$. If $R\left(k t_{n}\right) / 2 \leqslant R \leqslant R\left(k t_{n}\right)$, then $\lambda\left(t_{0}\right)=\lambda\left(k t_{n}\right)$ and by Lemma 4.2. $\lambda\left(k t_{n}\right) \leqslant C_{0} / R\left(t_{0}\right)$ and thus $u_{n}^{M}\left(\cdot, t_{0}\right) \leqslant h\left(\cdot, t_{0}\right)$. This and (4.1) imply that $h$ is a supersolution of $(\tilde{P})$ with $\lambda=\lambda\left(k t_{n}\right)$ on $\left(k t_{n},(k+1) t_{n}\right)$, and Theorem 3.7 leads to a contradiction.

Hence $R<R\left(k t_{n}\right) / 2$ and

$$
-\Delta u_{n}^{M}(\cdot, t)=\lambda\left(k t_{n}\right) \leqslant C_{0} / R \quad \text { for } t \in\left(k t_{n},(k+1) t_{n}\right] .
$$

Again Theorem 3.7 yields $u_{n}^{M} \leqslant \tilde{h}$ in $\mathbb{R}^{N} \times\left(k t_{n},(k+1) t_{n}\right]$, where

$$
\tilde{h}(x, t):=\left(R+A\left(t-k t_{n}\right)\right) f\left(x /\left(R+A\left(t-k t_{n}\right)\right)\right) .
$$

Since

$$
\{\tilde{h}(\cdot, t)>0\}=B_{R+A t}(0) \subset B_{R\left(k t_{n}\right)} \quad \text { on }\left[k t_{n},(k+1) t_{n}\right] \text { if } A t_{n} \leqslant R\left(k t_{n}\right) / 2,
$$

we obtain a contradiction for sufficiently small $n$.

REMARK Lemma 4.3 and the star-shapedness of $\Omega_{t}\left(u_{n}^{M}\right)$ imply that for each $t>0, \Omega_{t}\left(u_{n}^{M}\right)$ is a Lipschitz domain (i.e. its boundary is locally the graph of a Lipschitz function), whose Lipschitz constant is uniformly bounded for $0 \leqslant t \leqslant T$ independently of $n$ and $M$. This yields the following proposition:

Proposition 4.4 Suppose $u_{n}^{M}$ satisfies $(I)$ for $0 \leqslant t \leqslant T$ with $r=r(T)>0$. Then the distance function $d_{n}^{M}(\cdot, t)$ to $\Gamma\left(u_{n}^{M}\right)$ is locally uniformly Hölder continuous in time for $0 \leqslant t \leqslant T$, independently of $M$ and $n$.

Proof. Due to the previous lemma, $\Omega_{t}\left(u_{n}^{M}\right) \subset B_{R(t)}(0)$. Moreover, for $x_{0} \in \Gamma_{t_{0}}\left(u_{n}^{M}\right)$ with $t_{0} \in$ $\left(k t_{n},(k+1) t_{n}\right]$, there is a cone that touches the free boundary on which $u_{n}^{M}$ is zero:

$$
u_{n}^{M}\left(\cdot, t_{0}\right)=0 \quad \text { in } \mathcal{C}:=\left\{y: \frac{y-x_{0}}{\left|y-x_{0}\right|} \cdot \frac{x_{0}}{\left|x_{0}\right|} \geqslant \frac{\left|x_{0}\right|}{\sqrt{r^{2}+\left|x_{0}\right|^{2}}}\right\} .
$$


Let $B_{R}(0)$ be the smallest ball which contains $\Omega_{k t_{n}}\left(u_{n}^{M}\right)$. Due to Lemma $4.2 \lambda\left(k t_{n}\right) \leqslant C_{0} / R$. Moreover, arguing as in the proof of Lemma 4.3 it follows that $\Omega_{t}\left(u_{n}^{M}\right) \subset B_{R+A t}(0)$ for $t>k t_{n}$, where $A$ only depends on $r, V_{0}$ and $N$.

Note that, by $(I),\left|x_{0}\right| \geqslant r$. Fix $0<m \ll\left|x_{0}\right|, 1$ and let $g$ solve

$$
-\Delta g=C_{0} /(R-1) \quad \text { in } B_{R+1}(0)-\left(\mathcal{C}+m x_{0}\right),
$$

with $g=0$ on the boundary. Then for any $l>0$,

$$
\sup _{x \in B_{l}\left((1+m) x_{0}\right)} g(x) \leqslant C_{0} l^{\alpha}
$$

where $C_{0}=C_{0}(N)$ and $0<\alpha<1$ only depends on $R, r$ and $N$. Note that, since $\Omega_{t}\left(u_{n}^{M}\right)$ is star-shaped and the normal velocity of $\Gamma\left(u_{n}^{M}\right)$ is greater than $-1, \Omega_{t}\left(u_{n}^{M}\right)$ does not shrink more than distance $1 / A$ from $\Omega_{k t_{n}}\left(u_{n}^{M}\right)$ by $t=k t_{n}+1 / A$. Lemma 4.2 now yields, if we choose $A>1$,

$$
-\Delta u_{n}^{M}(\cdot, t) \leqslant C_{0} /(R-1) \quad \text { for } k t_{n} \leqslant t \leqslant k t_{n}+1 / A
$$

Due to (4.2), it follows that

$$
u_{n}^{M}(x, t) \leqslant g(x) \quad \text { for } k t_{n} \leqslant t \leqslant k t_{n}+1 / A
$$

as long as $u_{n}^{M}\left((1+m) x_{0}, t\right)=0$.

Next we construct a barrier $\phi(x, t)$ of the form

$$
\left\{\begin{array}{l}
-\Delta \phi(\cdot, t)=C_{0} / R \quad \text { in }\left(B_{2 m /\left|x_{0}\right|}-B_{r(t)}\right)\left((1+m) x_{0}\right), \\
\phi(\cdot, t)=0 \quad \text { on } \partial B_{r(t)}\left((1+m) x_{0}\right), \\
\phi(\cdot, t)=C_{0}\left(2 m /\left|x_{0}\right|\right)^{\alpha} \quad \text { on } \partial B_{2 m /\left|x_{0}\right|} \mid\left((1+m) x_{0}\right)
\end{array}\right.
$$

where $C_{0}$ is as given in (4.3) and

$$
r(t)=m /\left|x_{0}\right|-C_{1} m^{3 \alpha-3}\left(t-t_{0}\right) \quad \text { with } \quad C_{1}=c(N) C_{0}^{3}
$$

in the domain

$$
\mathcal{S}:=\left(B_{2 m /\left|x_{0}\right|}-B_{r(t)}\right)\left((1+m) x_{0}\right) \times\left[t_{0}, t_{1}\right], \quad t_{1}:=t_{0}+\left(2\left|x_{0}\right| C_{1}\right)^{-1} m^{4-3 \alpha} .
$$

It then follows that, on $\partial B_{r(t)}\left((1+m) x_{0}\right) \times\left[t_{0}, t_{1}\right], \phi$ satisfies

$$
\phi_{t} /|D \phi| \geqslant|D \phi|^{3}>|D \phi|^{3}-1
$$

if $c(N)$ is a sufficiently large dimensional constant. Due to (4.4), Theorem 3.7 applies to $u_{n}^{M}$ and $\phi$ in $\mathcal{S}$ as long as $u_{n}^{M}\left((m+1) x_{0}, t\right)=0$. But $u_{n}^{M}\left((m+1) x_{0}, t\right)=0$ as long as $u_{n}^{M} \leqslant \phi$. Thus we conclude that $u_{n}^{M} \leqslant \phi$ in $\mathcal{S}$.

In particular, the above argument shows that for any $m>0$, if $x_{0} \in \Gamma_{t_{0}}\left(u_{n}^{M}\right)$, then for any $m>0$, $\Gamma\left(u_{n}^{M}\right)$ does not reach $(1+m) x_{0}$ until $t_{1}=t_{0}+C(r, T, N) m^{4-3 \alpha}$. On the other hand, a parallel argument, based on the fact that $V=\left|D u_{n}^{M}\right|^{3}-1 \geqslant-1$, shows that $\Gamma\left(u_{n}^{M}\right)$ does not reach $(1-m) x_{0}$ until $t_{1}=t_{0}-m$. Since $m>0$ can be chosen arbitrarily small, we can conclude that $\Gamma\left(u_{n}^{M}\right)$ for $t \leqslant T$ is Hölder continuous in time with Hölder constant $1 /(4-3 \alpha)$, where $\alpha=\alpha(r, T, N)$. 
Let now $x$ be arbitrary. For times $t_{1}<t_{2}$, choose $x_{2} \in \Gamma_{t_{2}}\left(u_{n}^{M}\right)$ so that $\left|x-x_{2}\right|=d\left(x, t_{2}\right)$, and choose $x_{1}$ to be the unique point on $\Gamma_{t_{1}}\left(u_{n}^{M}\right)$ parallel to $x_{2}$. Using the Hölder continuity proved above, we have

$$
d\left(x, t_{1}\right) \leqslant\left|x-x_{1}\right| \leqslant\left|x_{2}-x_{1}\right|+\left|x-x_{2}\right| \leqslant C(r, T, N)\left|t_{2}-t_{1}\right|^{\alpha}+d\left(x, t_{2}\right)
$$

so that $d\left(x, t_{1}\right)-d\left(x, t_{2}\right) \leqslant C(r, T, N)\left|t_{2}-t_{1}\right|^{\alpha}$. We can analogously show $d\left(x, t_{2}\right)-d\left(x, t_{1}\right) \leqslant$ $C(r, T, N)\left|t_{2}-t_{1}\right|^{\alpha}$, which verifies uniform Hölder continuity.

We now prove the main result of this section.

THEOREM 4.5 (Existence of $u_{n}^{M}$ ) Suppose any weak solution $u_{n}^{M}$ of $(P)_{n}^{M}$ in $\mathbb{R}^{n} \times\left[0, t_{0}\right], t_{0} \leqslant T$, satisfies $(I)$ for $0 \leqslant t \leqslant t_{0}$, with $r=r(T)>0$. Then there exists a weak solution $u_{n}^{M}$ of $(P)_{n}^{M}$ with initial positive phase $\Omega_{0}$ and volume $V_{0}$ for $0 \leqslant t \leqslant T$. Moreover, $\Omega_{t}\left(u_{n}^{M}\right)$ is star-shaped with respect to $B_{r}(0)$ and $\Gamma_{t}\left(u_{n}^{M}\right)$ is locally uniformly Hölder continuous in time, independently of $n$ and $M$.

Proof. We use induction on $l$. Suppose we have constructed $u_{n}^{M}$ in $\mathbb{R}^{N} \times\left[0, l t_{n}\right]$. Due to Proposition 4.4. $\Omega_{t}\left(u_{n}^{M}\right)$ uniformly converges to $\Omega_{l t_{n}}\left(u_{n}^{M}\right)$ as $t \rightarrow l t_{n}$. Since $\Omega_{l t_{n}}\left(u_{n}^{M}\right)$ is starshaped, $\lambda\left(l t_{n}\right)$ and $u_{n}^{M}\left(\cdot, l t_{n}\right)$ are well-defined and continuous in space. Due to Theorem 3.8 there exists a viscosity solution $u_{n}^{M}$ of $(\tilde{P})$ with $\lambda=\lambda\left(l t_{n}\right)$ in $\left(l t_{n},(l+1) t_{n}\right]$ with initial positive phase $\Omega_{l t_{n}}\left(u_{n}^{M}\right)$. Now the induction can be continued to show that $u_{n}^{M}$ can be found for $0 \leqslant t \leqslant T$. The rest of the theorem is due to Lemma 3.9 and Proposition 4.4

\section{Convergence of $u_{n}^{M}$ and existence of $u^{M}$ and $u$}

In this section we prove the existence of the viscosity solution $u$ of our original problem $(P)$, by passing to limits in $n$ and $M$, and verifying that the result is a viscosity solution. First we fix $M$ and send $n \rightarrow \infty$. Due to Theorem 4.5 for $0 \leqslant t \leqslant T$ the signed distance function $d_{n}^{M}(\cdot, t)$ to the set $\Gamma_{t}\left(u_{n}^{M}\right)$ is locally uniformly Lipschitz continuous in space and locally uniformly Hölder continuous in time, independently of $n$ and $M$. Hence due to Arzelà-Ascoli, $d_{n}^{M}$ converges locally uniformly to $d^{M}$ in $\mathbb{R}^{n} \times[0, T]$ along a subsequence. It then follows that

(a) $\Omega_{t}^{M}:=\left\{d^{M}(\cdot, t)>0\right\}$ is star-shaped with respect to $B_{r}(0)$,

(b) $\Gamma_{t}^{M}:=\left\{d^{M}(\cdot, t)=0\right\}$, and the limiting distance function $d^{M}(\cdot, t)=0$ is locally Lipschitz in space and locally uniformly Hölder continuous in time,

(c) $\Omega_{0}^{M}=\Omega_{0}$.

Let $u^{M}(x, t)$ solve

$$
-\Delta u^{M}(\cdot, t)=\lambda\left(t ; u^{M}\right) \quad \text { in } \Omega_{t}^{M}
$$

with zero boundary data on $\Gamma_{t}^{M}$, where $\lambda\left(t ; u^{M}\right)$ is the volume preserving constant such that

$$
\int u^{M}(x, t) \mathrm{d} x=V_{0}
$$

Then $u^{M}(\cdot, 0)=u_{0}$.

PROPOSITION $5.1 u^{M}(x, t)$ is a viscosity solution of $(P)^{M}$ in $\mathbb{R}^{n} \times[0, T]$ with initial positive phase $\Omega_{0}$ and volume $V_{0}$. 
Proof. First observe that $\lambda_{n, M}\left(k t_{n}\right)$ converges to $\lambda\left(t ; u^{M}\right)$, locally uniformly in time, because $\Omega_{t}\left(u_{n}^{M}\right)$ locally uniformly converges to $\Omega_{t}\left(u^{M}\right)$ and $\Gamma_{t}\left(u_{n}^{M}\right)$ is locally uniformly Hölder continuous in time independently of $n$ (in the sense of the corresponding distance function). Therefore $u_{n}^{M}$ locally uniformly converges to $u^{M}$.

Now, suppose $u^{M}-\phi$ has a local maximum zero in $\bar{\Omega}\left(u^{M}\right) \cap\left(B_{r}\left(x_{0}\right) \times\left[t_{0}-r, t_{0}\right]\right)$ for some $r>0$ at $\left(x_{0}, t_{0}\right) \in \Gamma\left(u^{M}\right)$ with $\phi \in C^{2,1}(Q)$ and $|D \phi|\left(x_{0}, t_{0}\right) \neq 0$. Assume that, for some $\epsilon>0$,

$$
\left(\phi_{t} /|D \phi|-\min \left(|D \phi|^{3}-1, M\right)\right)\left(x_{0}, t_{0}\right)>\epsilon .
$$

We may assume that this maximum is strict in $B_{r}\left(x_{0}\right) \times\left[t_{0}-r, t_{0}\right]$ —otherwise one can replace $\phi$ by $\phi+\epsilon\left(x-x_{0}\right)^{4}+\epsilon\left(t-t_{0}\right)^{2}$ to make it strict. Since $\phi$ is smooth with $|D \phi|\left(x_{0}, t_{0}\right) \neq 0$, we may assume that a space-time ball of radius $r, B_{r}^{N+1}\left(P_{0}\right)$ with $P_{0} \in \mathbb{R}^{N+1}$, lies in the zero set of $u^{M}$ and touches $\Gamma\left(u^{M}\right)$ at $\left(x_{0}, t_{0}\right)$ (see Figure 2). Moreover due to (6.1) the outward normal vector $v$ of the ball $B_{r}^{N+1}\left(P_{0}\right)$ at $\left(x_{0}, t_{0}\right)$ is given by $v=\left(v_{1}, b\right) \in \mathbb{R}^{N} \times \mathbb{R}$, where $\left|v_{1}\right|=1$ and the slope $b$ of the ball at $\left(x_{0}, t_{0}\right)$ satisfies

$$
b \geqslant \min \left(|D \phi|^{3}-1, M\right)\left(x_{0}, t_{0}\right)+\epsilon .
$$

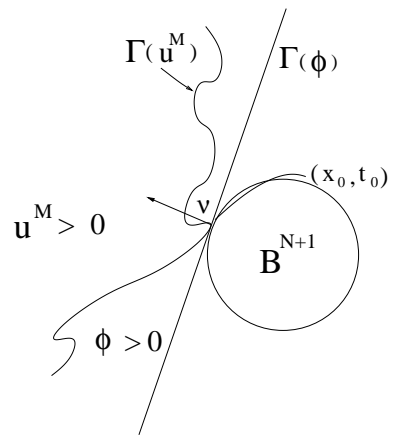

FIG. 2. Exterior ball $B_{r}^{N+1}\left(P_{0}\right)$ at the contact point $P_{0}$.

Let us tilt and shift the ball so that the new ball $\tilde{B}^{N+1}$ passes through $\left(x_{0}-a v_{1}, t_{0}\right)$ with slope $b-\epsilon / 2$ for $a \ll \epsilon$. Note that if $a$ is small compared to $\epsilon$ and $\tau$, then

$$
d\left(\tilde{B}^{N+1} \cap\left\{t=t_{0}-\tau\right\}, \Omega\left(u^{M}\right)\right)>O(\epsilon) .
$$

Now let us choose $a, \tau, \delta$ such that $a \ll \tau \ll \delta \ll r, \epsilon$ and define $h(x, t)$ in the domain

$$
\Sigma:=(1+a) \tilde{B}^{N+1} \cap\left[t_{0}-\tau, t_{0}\right]
$$

such that

$$
\left\{\begin{array}{l}
-\Delta_{x} h(x, t)=\lambda\left(t_{0}\right)+\delta \quad \text { in }\left((1+a) \tilde{B}^{N+1}-\tilde{B}^{N+1}\right) \cap\left[t_{0}-\tau, t_{0}\right], \\
h(x, t)=\phi(x, t) \quad \text { on }(1+a)\left(\partial \tilde{B}^{N+1}\right) \cap\left[t_{0}-\tau, t_{0}\right], \\
h(x, t)=0 \quad \text { in } \tilde{B}^{N+1} \cap\left[t_{0}-\tau, t_{0}\right] .
\end{array}\right.
$$

Since $\phi$ is smooth with $|D \phi|\left(x_{0}, t_{0}\right) \neq 0$, if $r$ is chosen small enough then (5.1) yields

$$
h_{t} /|D h|>\min \left(|D h|^{3}-1, M\right) \quad \text { on } \Gamma(h) \cap \Sigma .
$$


Moreover, due to (5.2), $u^{M} \prec h$ on the parabolic boundary of $\Sigma$. Since $u_{n}^{M}$ and $\Omega\left(u_{n}^{M}\right)$ locally uniformly converge to $u^{M}$ and $\Omega\left(u^{M}\right)$, it follows that $u_{n}^{M}$ crosses $h$ from below for the first time at $\left(y_{n}, s_{n}\right)$ in $\Sigma$ with $s_{n} \in\left(k t_{n},(k+1) t_{n}\right]$ for some $k \in \mathbb{N}$, for sufficiently large $n$. This contradicts Theorem 3.7 if $n$ is large enough that $\lambda_{n, M}\left(k t_{n}\right) \leqslant \lambda\left(t_{0}\right)+\delta$.

The above arguments prove that $u^{M}$ is a viscosity subsolution of $(P)^{M}$. A parallel argument would similarly prove that $u^{M}$ is a viscosity supersolution of $(P)^{M}$.

So far we have proved the existence of viscosity solutions of $(P)^{M}$. By a similar process, we can send $M \rightarrow \infty$ to obtain the most general existence result.

THEOREM 5.2 Suppose $u_{n}^{M}$ satisfies $(I)$ for $0 \leqslant t \leqslant T$ with $r=r(T)$. Then, along a subsequence, $u^{M}$ and $\Omega\left(u^{M}\right)$ locally uniformly converge to $u$ and $\Omega(u)$ in $\mathbb{R}^{n} \times[0, T]$ as $M \rightarrow \infty$. The limit function $u$ is a viscosity solution of $(P)$ in $\mathbb{R}^{n} \times[0, T]$ with initial positive phase $\Omega_{0}$ and volume $V_{0}$.

Corollary 5.3 Suppose $u_{n}^{M}$ satisfies $(I)$ for $0 \leqslant t \leqslant T$ with $r=r(T)$. Then there exists a viscosity solution $u$ of $(P)$ in $\mathbb{R}^{N} \times[0, T]$ with initial positive phase $\Omega_{0}$ and volume $V_{0}$. Moreover, $\Omega(u)$ is star-shaped in space with respect to $B_{r}(0)$, and $\Gamma(u)$ is Lipschitz continuous in space and Hölder continuous in time.

Since $\Omega(u)$ is only Lipschitz, difficulties arise in the analysis due to the lack of upper bound on the free boundary velocity. For this reason we will prove a uniqueness result for only the modified problem $(P)^{M}$ in the next section.

\section{Uniqueness of $u^{M}$}

In this section we show that $u^{M}$ given in Corollary 5.3 is the unique viscosity solution of $(P)^{M}$. Recall that $u_{n}^{M}$ is a weak solution of $(P)_{n}^{M}$ with initial positive phase $\Omega_{0}$ and volume $V_{0}$.

Proposition 6.1 Suppose $u_{n}^{M}$ satisfies $(I)$ for $0 \leqslant t \leqslant T$ with $r=r(T)$. Then for $k \geqslant n$ and $0 \leqslant t \leqslant T$, there exists $A>0$ depending only on $r, M, T$ and the spatial dimension $N$ such that

$$
A_{n}(t)^{-2 N-2} u_{k}^{M}\left(A_{n}(t) x, t\right) \leqslant u_{n}^{M}(x, t) \leqslant A(t)^{2 N+2} u_{k}^{M}\left(x / A_{n}(t), t\right),
$$

where $A_{n}(t):=1+A e^{A t} t_{n}$.

Proof. For simplicity we set $r=1 / 2$. A parallel argument holds for the general case.

Let $A=C_{0} M$, where $C_{0}$ is a sufficiently large constant which depends on $L$, the Lipschitz constant associated with the domain $\Omega_{l t_{n}}\left(u_{k}^{M}\right)$. Note that, due to Lemmas 3.9 and $4.2, L=$ $L(r, T, N)$.

For each $t \in[0,1 /((6 N+6) A)]$, we claim that

$$
\left(1+A_{n}\right)^{-2 N-2} u_{k}^{M}\left(\left(1+A_{n}\right) x, t\right) \prec u_{n}^{M}(x, t) \prec\left(1+A_{n}\right)^{2 N+2} u_{k}^{M}\left(\left(1+A_{n}\right)^{-1} x, t\right)
$$

where $A_{n}=A t_{n}$. At $t=0$ the inequality is true due to the star-shaped initial data. Suppose the second inequality in (6.1) is violated for the first time at $t=t_{0} \in(0,1 /((6 N+6) A)]$.

By (6.1),

$$
\left(1+A t_{n}\right)^{-1} \Omega_{t}\left(u_{k}^{M}\right) \subseteq \Omega_{t}\left(u_{n}^{M}\right) \subseteq\left(1+A t_{n}\right) \Omega_{t}\left(u_{k}^{M}\right) \text { for } 0 \leqslant t \leqslant t_{0} .
$$

Thus by definition of $\lambda_{n, M}$ and $\lambda_{k, M}$,

$$
\left(1+A t_{n}\right)^{-N} \lambda_{n, M}\left(l t_{n}\right) \leqslant \lambda_{k, M}\left(l t_{n}\right) \leqslant\left(1+A t_{n}\right)^{N} \lambda_{n, M}\left(l t_{n}\right)
$$

for any $l t_{n} \leqslant t_{0}, l=0,1, \ldots$ 
We claim that for any $x \in \Omega_{t}\left(u_{k}^{M}\right)$,

$$
d\left(\Omega_{l t_{n}}\left(u_{k}^{M}\right), x\right) \leqslant A t_{n} \quad \text { for } l t_{n} \leqslant t \leqslant(l+1) t_{n} .
$$

To verify (6.2), pick any point $x_{0}$ such that $d\left(\Omega_{l t_{n}}\left(u_{M}^{k}\right), x_{0}\right)=A t_{n}$. Since $\Omega_{l t_{n}}\left(u_{M}^{k}\right)$ is Lipschitz with Lipschitz constant $L$, there is a ball

$$
B:=B_{2 M}\left(x_{0}\right) \subset\left\{u_{k}^{M}\left(\cdot, l t_{n}\right)=0\right\}
$$

which touches $\Gamma_{l t_{n}}\left(u_{k}^{M}\right)$. Let us define

$$
\Lambda:=\sup _{(l-1) t_{n} \leqslant m t_{k} \leqslant(l+1) t_{n}} \lambda_{k, M}\left(m t_{k}\right) .
$$

Note that $\Lambda \leqslant r^{-N}$ by $(I)$.

Consider $\phi(x, t)$, a nonnegative function in $\Sigma:=3 B \times\left[l t_{n},(l+1) t_{n}\right]$ such that for $l t_{n} \leqslant t \leqslant$ $(l+1) t_{n}$,

$$
\left\{\begin{array}{l}
-\Delta \phi(\cdot, t)=\Lambda \quad \text { in } 3 B-\left(2-\frac{t-l t_{n}}{t_{n}}\right) B, \\
\phi(\cdot, t)=\sup _{\Sigma} u_{k}^{M} \text { on } \partial(3 B), \\
\phi(\cdot, t)=0 \quad \text { in }\left(2-\frac{t-l t_{n}}{t_{n}}\right) B .
\end{array}\right.
$$

Then $\phi$ is a supersolution of $(\tilde{P})$ in $\Sigma$ with $\lambda=\Lambda$ and $F(|D u|)=M$. Moreover, $u_{k}^{M} \prec \phi$ on the parabolic boundary of $\Sigma$, and $u_{k}^{M}(\cdot, t) \leqslant \phi(\cdot, t)$ as long as $\Omega_{t}\left(u_{k}^{M}\right) \cap 3 B \subset \Omega_{t}(\phi)$. Hence Theorem 3.7 applied to $u_{k}^{M}$ and $\phi$ in each time interval $\left(m t_{k},(m+1) t_{k}\right) \cap\left[l t_{n},(l+1) t_{n}\right]$ yields $u_{k}^{M} \leqslant \phi$ in $\Sigma$. In particular, $x_{0}$ lies outside of $\Omega\left(u_{k}^{M}\right)$ for $l t_{n} \leqslant t \leqslant(l+1) t_{n}$. This yields (6.2).

Due to (6.2),

$$
\left(1+A t_{n}\right)^{-2 N} \lambda_{k, M}\left(m t_{k}\right) \leqslant \lambda_{l, M}\left(l t_{n}\right) \leqslant\left(1+A t_{n}\right)^{2 N} \lambda_{k, M}\left(m t_{k}\right)
$$

for $m t_{k} \in\left[(l-1) t_{n},(l+1) t_{n}\right] \cap\left[0, t_{0}\right]$, where $m, l=0,1, \ldots$

Using (6.3) and Lemma 3.5, one can now check that for $0 \leqslant t \leqslant t_{0} \leqslant 1 /((6 N+6) A)$, the function

$$
\tilde{u}_{k}^{M}(x, t):=\left(1+A t_{n}\right)^{2 N+2} \inf _{x \in B_{A t_{n}-(6 N+6) A^{2} t_{n} t}} u_{k}^{M}\left(\left(1+A t_{n}\right)^{-1} x, t\right)
$$

satisfies the free boundary motion law

$$
\begin{aligned}
V & \geqslant \frac{\left(1+A t_{n}\right)\left(u_{k}^{M}\right)_{t}}{\left|D u_{k}^{M}\right|}+(6 N+6) A^{2} t_{n} \\
& =\left(1+A t_{n}\right) \min \left[\left(\left|D u_{k}^{M}\right|^{3}-1\right), M\right]+(6 N+6) A^{2} t_{n} \\
& \geqslant \min \left[\left(1+A t_{n}\right)^{6 N+1}\left|D u_{k}^{M}\right|^{3}-1, M\right]-(6 N+2) A t_{n} M+(6 N+6) A^{2} t_{n} \\
& \geqslant \min \left[\left|D \tilde{u}_{k}^{M}\right|^{3}-1, M\right]
\end{aligned}
$$

if $t_{n}$ is sufficiently small. The first inequality is due to Lemma 3.5 and the last inequality holds since $A>M$. (For a rigorous argument one needs to use the definition of viscosity solutions of $(\tilde{P})$. See for example the proof of Proposition 5.5 in [CJK].) 
Observe that due to (6.3) and Lemma 3.5 , for any $l, m=0,1, \ldots$

$$
\begin{aligned}
-\Delta \tilde{u}_{k}^{M}(\cdot, t) & \geqslant\left(1+A t_{n}\right)^{2 N} \lambda_{k, M}\left(m t_{k}\right) \\
& \geqslant \lambda\left(l t_{n}\right) \quad \text { for } t \in\left(m t_{k},(m+1) t_{k}\right] \cap\left[l t_{n},(l+1) t_{n}\right] .
\end{aligned}
$$

Note that $u_{n}^{M}(\cdot, 0) \prec \tilde{u}_{k}^{M}(\cdot, 0)$ since $\Omega_{0}$ is star-shaped with respect to zero and contains $B_{1}(0)$. Thus Theorem 3.7 applied to $u_{n}^{M}$ and $\tilde{u}_{n}^{M}$ on each time interval $\left[m t_{k},(m+1) t_{k}\right]$ gives for $0 \leqslant t \leqslant t_{0}$,

$$
u_{n}^{M}(\cdot, t) \prec \tilde{u}_{k}^{M}(\cdot, t) \leqslant\left(1+A t_{n}\right)^{2 N+2} u_{k}^{M}\left(\left(1+A t_{n}\right)^{-1} x, t\right) .
$$

This contradicts our hypothesis at $t=t_{0}$. Similar arguments lead to a contradiction if we assume that the first inequality breaks down the first time at $t_{1} \in[0,1 /((6 N+6) A)]$. Thus $(6.1)$ holds for $0 \leqslant t \leqslant t_{1}:=1 /((6 N+6) A)$

Next we show that for $t_{1} \leqslant t \leqslant t_{1}(1+1 / 2)$,

$$
\left(1+2 A t_{n}\right)^{-2 N-2} u_{k}^{M}\left(\left(1+2 A t_{n}\right) x, t\right) \leqslant u_{n}^{M}(x, t) \leqslant\left(1+2 A t_{n}\right)^{2 N+2} u_{k}^{M}\left(\left(1+2 A t_{n}\right)^{-1} x, t\right) .
$$

For example if the second inequality breaks down, then we compare $u_{n}^{M}$ with

$$
\tilde{u}_{k}^{M}(x, t):=\left(1+2 A t_{n}\right)^{2 N+2} \inf _{x \in B_{A t_{n}-12(N+1) A^{2} t_{n} t}} u_{k}^{M}\left(\left(1+2 A t_{n}\right)^{-1} x, t\right)
$$

using similar arguments as for (6.1). Note that due to (6.1) and the fact that $\Omega_{t}\left(u_{n}\right)$ is star-shaped and contains $B_{1}(0)$,

$$
u_{n}^{M}\left(x, t_{1}\right) \prec\left(1+A t_{n}\right)^{2 N+2} u_{k}^{M}\left(\left(1+A t_{n}\right)^{-1} x, t_{1}\right) \leqslant \tilde{u}_{k}^{M}\left(x, t_{1}\right) .
$$

One can repeat the argument for each interval

$$
\left[t_{1}(1+1 / 2+\cdots+1 / n), t_{1}(1+1 / 2+\cdots+1 / n+1)\right] \text {. }
$$

This proves the lemma since

$$
t_{1}(1+1 / 2+\cdots+1 / n) \sim t_{1}(\log n) .
$$

Note that the proof presented above can be used as long as one of the functions being compared, $u_{k}^{M}$ in the above proof, satisfies $(I)$. Thus we obtain the following corollary.

Corollary 6.2 Suppose $u_{n}^{M}$ satisfies $(I)$ for $0 \leqslant t \leqslant T$ with $r=r(T)$. Then the whole sequence $\left\{u_{n}^{M}\right\}$ converges locally uniformly as $n \rightarrow \infty$ to a viscosity solution $u^{M}$ of $(P)^{M}$ for $0 \leqslant t \leqslant T$ with initial positive phase $\Omega_{0}$ and volume $V_{0}$.

REMARKS 1. Besides proving the uniqueness of the limit, Proposition 6.1 provides an estimate on differences between discrete-time approximation solutions $u_{n}^{M}$ in terms of the discrete time interval size $t_{n}$.

2. Note that we need to keep track of both inequalities in the lemma in each time interval to guarantee that $\lambda_{n, M}(t)$ and $\lambda_{k, M}(t)$ stay close together.

Now let $v$ be any other viscosity solution with initial data $u_{0}$ defined in the previous section. Parallel arguments to the proof of Proposition 6.1 yield the following: 
Lemma 6.3 Suppose $u_{n}^{M}$ satisfies ( $\left.I\right)$ for $0 \leqslant t \leqslant T$ with $r=r(T)$. Then for the same $A_{n}(t)$ given in Proposition 6.1.

$$
\left(1+A_{n}(t)\right)^{-2 N-2} u_{n}^{M}\left(\left(1+A_{n}(t)\right) x, t\right) \leqslant v(x, t) \leqslant\left(1+A_{n}(t)\right)^{2 N+2} u_{n}^{M}\left(\frac{x}{1+A_{n}(t)}, t\right)
$$

for $0 \leqslant t \leqslant T$.

Applying the same argument for two viscosity solutions $u^{M}$ and $v^{M}$ of $(P)^{M}$ (in this case the time step size $t_{n}>0$ is replaced by arbitrary small constants in the arguments ) yields the following corollary.

COROLlaRY 6.4 Suppose $u_{n}^{M}$ satisfies $(I)$ for $0 \leqslant t \leqslant T$ with $r=r(T)$. Then $u^{M}$ is the unique viscosity solution of $(P)^{M}$ in $\mathbb{R}^{n} \times[0, T]$ with initial data $u_{0}$.

REMARK To prove uniqueness results for the original problem $(P)$, one needs some type of bound on free boundary velocity. At least for star-shaped spreading droplets, we expect solutions of $(P)$ to have smooth positive phase for positive times and locally uniformly bounded free boundary velocity for any positive time interval. Such results have been proved for the Hele-Shaw problem with zero surface tension (see $[\mathrm{CJK}])$.

\section{Appendix A. Comparison principle and existence for $(\tilde{P})$}

Here we prove Theorem 3.7 and the existence of the viscosity solutions of $(\tilde{P})$ with star-shaped initial positive phase $\Omega_{0}$.

Most arguments presented here are similar to the proofs of Theorem 2.2 and Theorem 4.7 of [K1]. We only sketch the proof below.

Sketch of the proof of Theorem 3.7 For $r, \delta>0$ and $0<h \ll r$, define the sup-convolution of $u$

$$
Z(x, t):=(1+\delta) \sup _{|(y, s)-(x, t)|<r} u\left(y,(1+\delta)^{3} s\right)
$$

and the inf-convolution of $v$

$$
W(x, t):=(1-\delta) \inf _{|(y, s)-(x, t)|<r-h t} v\left(y,(1-\delta)^{3} s\right)
$$

in the domain

$$
\Sigma:=\tilde{D} \times[r, r / h], \quad \tilde{D}:=\{x: x \in D, d(x, \partial D) \geqslant r\} .
$$

By upper semicontinuity of $u-v, Z(\cdot, r) \prec W(\cdot, r)$ for sufficiently small $r, \delta>0$. By our hypothesis and the upper semicontinuity of $u-v$,

$$
Z(\cdot, t) \prec W(\cdot, t) \quad \text { on } \partial \tilde{D} \text { for } r \leqslant t \leqslant r / h
$$

for sufficiently small $\delta$ and $r$. Moreover, Lemma 3.5 shows that $Z$ and $W$ are respectively sub- and supersolutions of $(\tilde{P})$ in $\tilde{D} \times[r, r / h]$.

If our theorem is not true for $u$ and $v$, then $Z$ crosses $W$ from below for the first time at $P_{0}:=$ $\left(x_{0}, t_{0}\right) \in \tilde{D} \times[r, r / h]$ for $h \ll r$. Due to the maximum principle of harmonic functions and 


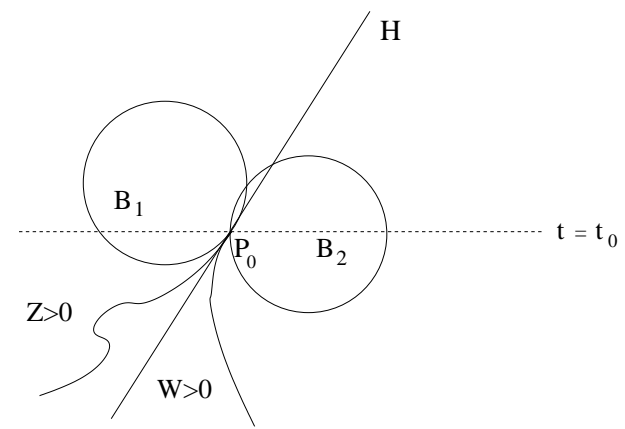

FIG. A. Interior and exterior balls at the contact point $P_{0}$.

Lemma 3.4, $P_{0} \in \Gamma(Z) \cap \Gamma(W)$. Note that by definition $\Omega(Z)$ and $\Omega(W)$ have respectively an interior ball $B_{1}$ and exterior ball $B_{2}$ at $P_{0}$ of radius $r$ in space-time (see Figure 3).

Let us denote by $H$ the tangent hyperplane to the interior ball of $Z$ at $P_{0}$. Since $Z \leqslant W$ for $t \leqslant t_{0}$ and $P_{0} \in \Gamma(Z) \cap \Gamma(W)$, it follows that

$$
B_{1} \cap\left\{t \leqslant t_{0}\right\} \subset \Omega(Z) \cap \Omega(W), \quad B_{2} \cap\left\{t \leqslant t_{0}\right\} \subset\{Z=0\} \cap\{W=0\}
$$

with $\bar{B}_{1} \cap \bar{B}_{2} \cap\left\{t \leqslant t_{0}\right\}=\left\{P_{0}\right\}$.

Moreover, by Lemma 3.5 .

$$
\frac{Z_{t}}{|D Z|}(x, t) \leqslant F(|D Z|)(x, t) \quad \text { on } \Gamma(Z)
$$

and

$$
\frac{W_{t}}{|D W|}(x, t) \geqslant F(|D W|)(x, t)+h \quad \text { on } \Gamma(W) .
$$

In particular, by (A.1) the argument of Lemma 2.5 in [K1] applies for $Z$ to show that $H$ is not horizontal. In particular, $B_{1} \cap\left\{t=t_{0}\right\}$ and $B_{2} \cap\left\{t=t_{0}\right\}$ share the same normal vector $v_{0}$, outward with respect to $B_{1}$, at $P_{0}$.

Formally speaking, it follows that $\frac{Z_{t}}{|D Z|}\left(x_{0}, t_{0}\right)<\infty$ and

$$
\frac{Z_{t}}{|D Z|}\left(P_{0}\right) \leqslant F(|D Z|)\left(P_{0}\right) \leqslant F(|D W|)\left(P_{0}\right) \leqslant \frac{W_{t}}{|D W|}\left(P_{0}\right)-h,
$$

where the second inequality follows since $F(r)$ is increasing in $r$ and $Z\left(\cdot, t_{0}\right) \leqslant W\left(\cdot, t_{0}\right)$ in a neighborhood of $x_{0}$. The above inequality says that the free boundary speed of $Z$ is strictly less than that of $W$ at $P_{0}$, contradicting the fact that $\Gamma(Z)$ touches $\Gamma(W)$ from below at $P_{0}$.

For a rigorous argument one can construct radially symmetric barrier functions based on the exterior and interior ball properties of $Z$ and $W$ at $P_{0}$ to derive a version of (A.1) and yield a contradiction. For details see the proof of Theorem 2.2 in [K1].

Next we prove Theorem 3.8

Proof of Theorem 3.8. We apply Perron's method. Without loss of generality we assume that $F(r) \geqslant F(0) \geqslant-1$. Since $\Omega_{0}$ is star-shaped with respect to $B_{h}(0)$, there exist $C>0$ and $0<\alpha<1$ 
such that for any $r>0$ and $x_{0} \in \Gamma_{0}$,

$$
\sup _{x \in B_{r}\left(x_{0}\right)} u_{0}(x) \leqslant C r^{\alpha} .
$$

Let us define

$$
U_{1}(x, t):= \begin{cases}\left(1-t / h^{2}\right)^{2} u_{0}\left(\frac{x}{1-t / h^{2}}\right) & \text { for } 0 \leqslant t \leqslant h^{2} \\ 0 & \text { for } t \geqslant h^{2},\end{cases}
$$

and

$$
U_{2, r}(x, t):=(1+r)^{2} \inf _{y \in B_{r(t)}(x)} u_{0}\left((1+r)^{-1} y\right),
$$

where $r(t):=r-C_{1} r^{\alpha-1} t$ for $0 \leqslant t \leqslant\left(C_{1}\right)^{-1} r^{\alpha}$. Note that due to (A.3), $U_{2, r}$ is a supersolution of $(\tilde{P})$ for sufficiently large $C_{1}$. Moreover $U_{1}(x, t)$ is a subsolution of $(\tilde{P})$ since $F \geqslant-1$ and $\Omega\left(u_{0}\right)$ contains $B_{h}(0)$.

Let $z \in \mathcal{P}$ if and only if $z(x, t)$ is a viscosity subsolution of $(\tilde{P})$ with $z(\cdot, 0) \leqslant u_{0}(x)$ and $U_{1} \leqslant z$ in $\mathbb{R}^{N} \times[0, \infty)$. Let

$$
U(x, t):=\sup \{z(x, t): z \in \mathcal{P}\}
$$

Arguing as in the proof of Theorem 4.7 in [K1] shows that $U^{*}$ and $U_{*}$ are respectively viscosity subsolution and supersolution of $(\tilde{P})$. Moreover, by Theorem 3.7. $U^{*} \leqslant U_{2, r}$ for $0 \leqslant t \leqslant r^{\alpha}$ for any $r>0$. In particular, $U_{*}=U^{*}=u_{0}$ at $t=0$. In other words, $U_{*}$ is a viscosity solution of $(\tilde{P})$.

\section{Appendix B. Global-time existence and uniqueness for solutions with symmetry}

The purpose of this section is to illustrate some examples where $u_{n}^{M}$ satisfies $(I)$ for all $t \geqslant 0$ with $r=r(t)$. For simplicity, we set $\int_{\Omega_{0}} u=1$ and $\Omega_{t}(u) \subset B_{1}(0)$.

\section{B.1 Reflection comparison}

LEMMA B.1 (Strong comparison principle) Let $u, v$ be resp. viscosity sub- and supersolutions of $(\tilde{P})$ in $\Sigma=D \times(a, b)$ with $u \leqslant v$ at $t=a$ and on $\partial D \times(a, b)$. In addition suppose that $u$ satisfies (I) for $a \leqslant t \leqslant b$. Then $u(\cdot, t) \leqslant v(\cdot, t)$ in $D$ for $a<t<b$.

Proof. For simplicity let $a=0$. Let us define

$$
\tilde{u}(x, t):=(1+\epsilon)^{-2} \sup _{y \in B_{r \epsilon}(x)} u((1+\epsilon) y, t) .
$$

Observe that $\tilde{u}$ is a subsolution of $(\tilde{P})$ by Lemma 3.5. Also observe that, since $\Omega_{t}(u)$ is star-shaped with respect to $B_{r}(0)$ and $-\Delta u=\lambda$ in $\Omega(u)$,

$$
\tilde{u}(x, t) \prec v(x, t) \quad \text { on the parabolic boundary of } \Sigma .
$$

Hence Theorem 3.7 shows that $\tilde{u} \prec v$ for $0 \leqslant t \leqslant b$ for any $\epsilon>0$, and thus $u \leqslant v$.

Recall that $u_{n}^{M}$ solves $(P)_{n}^{M}$ with given initial positive phase $\Omega_{0}\left(u_{n}^{M}\right)=\Omega_{0}$ (see Definition 4.1). 
LEMMA B. 2 Suppose $u_{n}^{M}$ satisfies $(I)$ for $0 \leqslant t \leqslant T$. Let $H$ be any hyperplane in $\mathbb{R}^{n}$ and let $\phi_{H}(x)$ be the reflection of $x$ with respect to $H$. Let $D_{1}$ and $D_{2}$ be the half-spaces in $\mathbb{R}^{n}$ determined by $H$. If

$$
u_{n}^{M}\left(x, t_{0}\right) \leqslant u_{n}^{M}\left(\phi_{H}(x), t_{0}\right) \quad \text { in } D_{1}
$$

then

$$
u_{n}^{M}(x, t) \leqslant u_{n}^{M}\left(\phi_{H}(x), t\right) \quad \text { in } D_{1} \text { for } t_{0}<t \leqslant T .
$$

Proof. Set $v(x, t):=u_{n}^{M}\left(\phi_{H}(x), t\right)$. Then $v$ solves the following equation in $\left(k t_{n},(k+1) t_{n}\right]$, $k=1,2, \ldots$ :

$$
\left\{\begin{array}{l}
-\Delta v(\cdot, t)=\lambda_{n}^{M}\left(k t_{n}\right) \quad \text { in } \Omega(v), \\
v_{t}=|D v| \min \left(\left(|D v|^{3}-1\right), M\right) \text { on } \Gamma(v) .
\end{array}\right.
$$

Moreover, $v=u_{n}^{M}$ on $H=\partial D_{1}=\partial D_{2}$. Since $u_{n}^{M}$ has a compact support in any finite time period (Lemma 4.3), Lemma B.1 applies to $u_{n}^{M} \leqslant v$ in $D_{1}$ in $\left(k t_{n},(k+1) t_{n}\right.$ ] for $t_{0}<t \leqslant T$.

Corollary B.3 Suppose that $\Omega_{0}\left(u_{n}^{M}\right) \subset B_{R}(0), R>1$ and $u_{n}^{M}$ satisfies $(I)$ for $0 \leqslant t \leqslant T$. Then $\Omega_{t}\left(u_{n}^{M}\right) \subset B_{3 R}(0)$ for $0 \leqslant t \leqslant T$.

Proof. For $x_{0} \in B_{3 R}(0)-B_{2 R}(0)$, define

$$
\mathcal{C}\left(x_{0}\right):=\left\{y: y \cdot x_{0} \leqslant-\frac{1}{2}\left|x_{0}\right||y|\right\} .
$$

If we pick a hyperplane $H$ normal to a vector $y_{0} \in \mathcal{C}\left(x_{0}\right)$ containing $x_{0}$, then

$$
u_{n}^{M}\left(\phi_{H}(x), 0\right)=0 \leqslant u_{n}^{M}(x, 0) \quad \text { in } D_{1}:=\left\{t y_{0}+h: t>0, h \in H\right\} .
$$

Hence Lemma B.1 shows that $u_{n}^{M}$ is increasing in the cone of directions $\mathcal{C}\left(x_{0}\right)$.

Suppose $\Gamma_{t}\left(u_{n}^{n}\right)$ touches $\partial B_{3 R}(0)$ for the first time at $x_{0}$ at $t=t_{0}$. Then (B.1) yields

$$
\left(x_{0}+\mathcal{C}\left(x_{0}\right)\right) \cap\left(B_{3 R}(0)-B_{2 R}(0)\right) \subset \Omega_{t_{0}}(u) .
$$

Since $\int u_{n}^{M}\left(\cdot, k t_{n}\right) \mathrm{d} x=1$, we obtain $\lambda \leqslant R^{-n-2}$. On the other hand, $u_{n}^{M}\left(\cdot, t_{0}\right)=0$ outside of $B_{3 R}(0)$. Now comparing $u_{n}^{M}\left(\cdot, t_{0}\right)$ with $f(x):=(3 R)^{-n}-(3 R)^{-n-2} x^{2}$ yields $\left|D u_{n}^{M}\right|\left(x_{0}, t_{0}\right)<1$. Therefore the outward normal velocity of $\Gamma\left(u_{n}^{M}\right)$ at $\left(x_{0}, t_{0}\right)$ is strictly negative, contradicting the definition of $t_{1}$.

For $B_{r}(x) \subset \Omega_{0}$, let

$$
t(x, r):=\sup \left\{t: B_{r}(x) \subset \Omega_{t}\left(u_{n}^{M}\right)\right\} .
$$

Note that, due to Lemma 3.4, $B_{r}(x)$ is touched by $\Gamma_{t}(u)$ for the first time at $t=t(x, r)$.

LEMMA B.4 Suppose $u_{n}^{M}$ satisfies $(I)$ for $0 \leqslant t \leqslant T$. Suppose $\Omega_{0}(u)$ is star-shaped with respect to $B_{r}\left(x_{0}\right)$ with $t_{0}:=t\left(x_{0}, r\right) \leqslant T$. Let $y \in \partial B_{r}\left(x_{0}\right) \cap \Gamma_{t_{0}}(u)$ and let $H$ be the hyperplane normal to $y-x_{0}$ containing $x_{0}$. Then

$$
u_{n}^{M}\left(\phi_{H}(x), t\right) \leqslant u_{n}^{M}(x, t) \quad \text { in } D_{1} \times\left[t_{0}, T\right],
$$

where $D_{1}$ is the half-space determined by $H$ containing $B_{r}\left(x_{0}\right)$.

Proof. Note that, since $\Omega_{t_{0}}\left(u_{n}^{M}\right)$ is star-shaped with respect to $B_{r}\left(x_{0}\right)$ with $y_{0} \in \partial B_{r}\left(x_{0}\right) \cap \Gamma_{t_{0}}\left(u_{n}^{M}\right)$,

$$
u_{n}^{M}\left(\phi_{H}(x), t_{0}\right)=0 \quad \text { in } D_{1} .
$$

Now we can conclude by applying Lemma B.2. 
B.2 Example 1: Two symmetric axes

Let $e_{1}, \ldots, e_{n}$ be an orthonormal basis in $\mathbb{R}^{n}$.

THEOREM B.5 Suppose $\Omega_{0}$ is star-shaped with respect to $B_{r}(0)$ and is symmetric with respect to the $e_{1}$-axis and $e_{2}$-axis. Then for any $T>0, n$ and $M, u_{n}^{M}$ satisfies $(I)$ with $r=r(T)>0$ for $0 \leqslant t<T$.

REMARK Due to Lemma B.2, $\Omega_{t}\left(u_{n}^{M}\right)$ stays symmetric with respect to the $e_{1}$-axis and $e_{2}$-axis.

Proof. Define $t_{0}:=t(0, r)>0$. If $t_{0}=\infty$ then we are done, so suppose $t_{0}$ is finite. Then $B_{r}(0) \subset$ $\Omega\left(u_{0}\right)$ and $\Gamma_{t_{0}}\left(u_{n}^{M}\right)$ touches $\bar{B}_{r}(0)$ at some point $x_{0} \in \partial B_{r}(0)$.

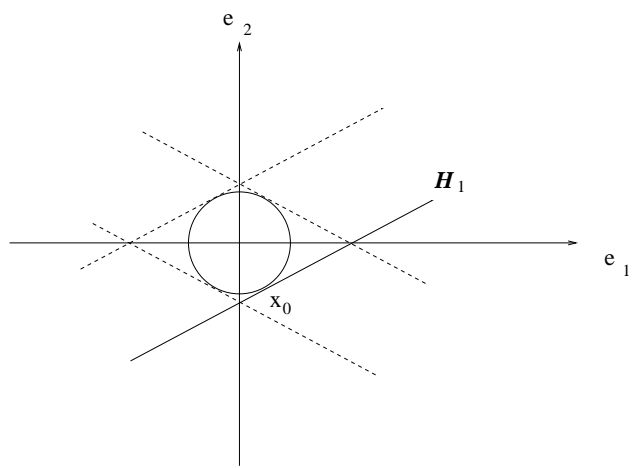

FIG. 4. Parallel hyperplanes bounding $\Omega_{t}\left(u_{n}^{M}\right)$

Let $v:=-x_{0}$ and let $H$ be the hyperplane which is orthogonal to $v$. Let $D_{1}$ be the half-space bounded by $H$ which does not contain $B_{r}(0)$. Note that up to $t=t_{0}, \Omega_{t}\left(u_{n}^{M}\right)$ is star-shaped with respect to $B_{r}(0)$. Hence $u_{n}^{M}\left(\cdot, t_{0}\right)=0$ in $D_{1}$. By symmetry, $u_{n}^{M}\left(\cdot, t_{0}\right)=0$ in the reflected image of $D_{1}$ with respect to the $e_{1}$-axis and $e_{2}$-axis. Thus $\Omega_{t_{0}}\left(u_{n}^{M}\right)$ lies between two parallel hyperplanes with distance at most $2 r$ (see Figure 4). Recall that due to Corollary B.3, $\Omega_{t}\left(u_{n}^{M}\right) \subset B_{R}(0)$ for some $R>0$. Thus it follows that

$$
\operatorname{Vol}\left(\Omega_{t_{0}}\left(u_{n}^{M}\right)\right) \leqslant C(n) R^{n-1} r .
$$

Recall that $\Omega_{t_{0}}\left(u_{n}^{M}\right)=0$ in $D_{1}$. If we choose $r$ sufficiently small it follows that $\left|D u_{n}^{M}\right|\left(x_{0}, t_{0}\right)>1$ (a detailed argument is given in the proof of Theorem B.7, Case 1). This means that $\Omega\left(u_{n}^{M}\right)$ is strictly expanding at $\left(x_{0}, t_{0}\right)$, contradicting the definition of $t_{0}$.

The above theorem in particular states that if a droplet with two symmetry axes satisfies $(I)$ initially, then it never changes its topology at a later time, however thin and long it is. On the contrary, we will show below that a dumbbell-shaped droplet changes its topology in finite time.

LEMMA B.6 Suppose $u_{n}^{M}$ solves $(P)_{n}^{M}$ with initial positive phase

$$
\Omega_{0}:=B_{1}\left(-3 e_{1}\right) \cup B_{1}\left(3 e_{1}\right) \cup\left\{x=\left(x_{1}, x^{\prime}\right):\left|x^{\prime}\right| \leqslant r,\left|x_{1}\right| \leqslant 3\right\} .
$$

If $r$ is smaller than a dimensional constant, then $\Omega\left(u_{n}^{M}\right)$ changes its topology before $t=1 / 2$. 
Proof. First observe that, since the free boundary velocity is greater than -1 , for $0 \leqslant t \leqslant 1 / 2$ we have $B_{1 / 2}\left( \pm 3 e_{1}\right) \subset \Omega_{t}\left(u_{n}^{M}\right)$. Hence $\lambda\left(u_{n}^{M} ; t\right)<C(n)$ for $0 \leqslant t \leqslant 1 / 2$ and some $C(n) \geqslant 1$. Pick $T=T(n)$ sufficiently small so that $\Omega_{t}\left(u_{n}^{M}\right) \subset B_{10}(0)$ for $0 \leqslant t \leqslant T$. Now one can compare $u_{n}^{M}$ with

$$
h(x, t):=C(n) \min \left[\left(|x|^{2}-100\right)_{+},\left(r(t) x_{1}^{4}-3^{4}\left|x^{\prime}\right|^{2}+3^{4} r^{2}\right)_{+}\right]
$$

where $r(t):=\left(1-(10 C(n))^{3} t\right)^{-1 / 3}$ for $0 \leqslant t \leqslant t_{0}:=(10 C(n))^{-4}$. One can choose $C(n)$ sufficiently large such that $t_{0} \leqslant T$.

Observe that $\Omega\left(u_{0}\right) \subset \Omega_{0}(h)$ and $-\Delta h(\cdot, t) \geqslant C(n)$. Also a straightforward computation yields

$$
h_{t}=4 C(n) r^{\prime}(t) x_{1}^{3} \geqslant|D h|\left(-1 / 2+|D h|^{3}\right) \quad \text { on } \Gamma_{t}(h)
$$

for $0 \leqslant t \leqslant t_{0}$, if $0 \leqslant r \leqslant 4^{-4}$.

Hence if we set

$$
\tilde{h}(x, t):=\inf _{y \in B_{t / 2}(x)} h(y, t),
$$

then $\tilde{h}$ is a supersolution of $(\tilde{P})$ with $\lambda=C(n)$. Now Theorem 3.7 yields $u_{n}^{M} \prec \tilde{h}$ in $\mathbb{R}^{n} \times\left[0, t_{0}\right]$.

If $r \leqslant \frac{1}{2} t_{0}$ then it follows that $\Omega_{t_{0}}(\tilde{h})$ is no longer simply connected, and therefore neither is $\Omega_{t_{0}}\left(u_{n}^{M}\right)$ (a change of topology occurred before $t=t_{0} \leqslant 1 / 2$.)

\section{B.3 Example 2: One axis symmetry with convexity}

Here we set the dimension $N=2$.

TheOREM B.7 Suppose $\Omega_{0} \subset \mathbb{R}^{2}$ is convex and symmetric with respect to the $e_{1}$-axis. Then for any $T>0, n$ and $M, u_{n}^{M}$ satisfies $(I)$ with $r=r(T)>0$ for $0 \leqslant t \leqslant T$.

Proof. Let

$$
\mathcal{S}:=\left\{B_{r}(y) \subset \bar{\Omega}_{0}: y \in \Omega_{0} \cap\left\{x=\left(x_{1}, 0, \ldots, 0\right)\right\} .\right.
$$

Then for each ball in $\mathcal{S}$ there is the first time $\Gamma_{t}\left(u_{n}^{M}\right)$ touches the ball. Let $t_{0}$ be the supremum of these times. Then $\Gamma_{t_{0}}\left(u_{n}^{M}\right)$ touches $y_{0} \in \partial B_{r}\left(x_{0}\right)$ for some $B_{r}\left(x_{0}\right) \subset \mathcal{S}$. We may assume that $\left(y_{0}-x_{0}\right) \cdot e_{1} \leqslant 0$. Let $l_{0}$ be the line normal to $y_{0}-x_{0}$ with $y_{0} \in l$.

First assume that $B_{r}\left(x_{1}\right) \subset \Omega_{0}$, where $x_{1}:=x_{0}+r^{1 / 2} e_{1}$. Then when the free boundary hits the boundary of $B_{r}\left(x_{1}\right)$ for the first time at $t=t_{1} \leqslant t_{0}$ it does not cross $B_{r}\left(x_{0}\right)$. Therefore the first touching point $y_{1} \in \partial B_{r}\left(x_{1}\right)$ satisfies $\left(y_{1}-x_{1}\right) \cdot e_{1} \geqslant 0$. Let $l_{1}$ be the line normal to $y_{1}-x_{1}$ with $y_{1} \in l_{1}$ and let $e_{1}$ point to the right, horizontally.

By the above, $u_{n}^{M}\left(\cdot, t_{0}\right)=0$ on the left side of $l_{0}$. Moreover, $u_{n}^{M}\left(\cdot, t_{1}\right)=0$ on the right side of $l_{1}$. By symmetry, $u_{n}^{M}\left(\cdot, t_{1}\right)=0$ on the right side of $\tilde{l}_{1}$, the reflection of $l_{1}$ with respect to the $e_{1}$-axis (see Figure 5). Let $\theta_{0}$ be the angle between $l_{0}$ and $e_{1}$, and $\theta_{1}$ be the angle between $l_{1}$ and $e_{1}$.

Case 1: $\theta_{1}<r^{1 / 2}$. By the above argument $u_{n}^{M}\left(\cdot, t_{1}\right)=0$ outside of the cone of angle $r^{1 / 2}$ along the $e_{1}$-axis. Since $\Omega_{t}\left(u_{n}^{M}\right) \subset B_{R}(0)$ for some $R, \Omega_{t}\left(u_{n}^{M}\right)$ is contained in a cone of angle $r^{1 / 2}$ and height $6 R$. Let $\lambda_{0}:=\lambda\left(t_{1} ; u_{n}^{M}\right)=\lambda_{0}$ and

$$
h(x):=\frac{\lambda_{0}}{2}\left(\left[3 R r^{1 / 2}\right]^{2}-\left(x_{2}\right)^{2}\right) .
$$

Since $-\Delta h=\lambda_{0}$ and

$$
\Omega_{t_{1}}\left(u_{n}^{M}\right) \subset \Omega(h)=\left\{x:\left|x_{2}\right| \leqslant 3 R r^{1 / 2}\right\},
$$




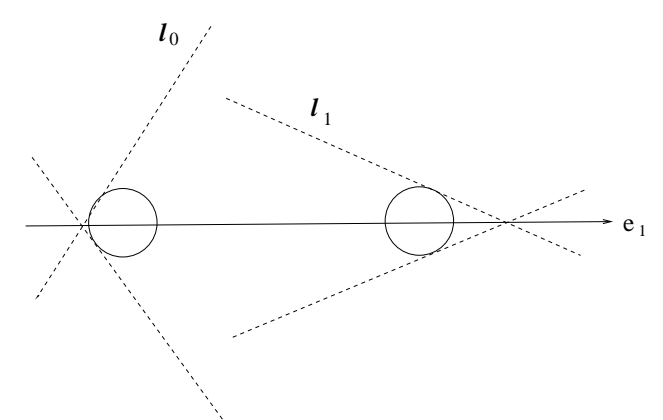

FIG. E. The lines $l_{0}, l_{1}$ and their reflections.

we have $u_{n}^{M} \leqslant h$. Thus

$$
\int u_{n}^{M}\left(\cdot, t_{1}\right) \mathrm{d} x \leqslant \int_{\Omega_{t_{1}}\left(u_{n}^{M}\right)} h(x) \mathrm{d} x \leqslant\left(\lambda_{0} R^{2} r\right) \operatorname{Vol}\left(\Omega_{t_{1}}\left(u_{n}^{M}\right)\right) \leqslant \lambda_{0}\left(3 R r^{1 / 2}\right)^{3} .
$$

Since $\int u_{n}^{M}\left(\cdot, k t_{n}\right) \mathrm{d} x=1$, we obtain $\lambda_{0} \geqslant\left(3 R r^{1 / 2}\right)^{-3}$ if $r<(3 R)^{-6}$. Note that $B_{r}\left(x_{1}\right) \subset$ $\Omega_{t_{1}}\left(u_{n}^{M}\right)$, and thus

$$
u_{n}^{M}\left(\cdot, t_{1}\right) \geqslant f(x)=(3 R)^{-3} r^{1 / 2}-\frac{\left(3 R r^{1 / 2}\right)^{-3}}{2}\left(x-x_{1}\right)^{2} .
$$

In particular, $\left|D u_{n}^{M}\right|\left(y_{1}, t_{1}\right) \geqslant|D f|\left(y_{1}\right)=(3 R)^{-3} r^{-1 / 2}>1$ if $r<(3 R)^{-6}$. This contradicts the fact that the outward normal velocity of $\Gamma\left(u_{n}^{M}\right)$ at $\left(x_{1}, t_{1}\right)$ is nonnegative.

Case 2: $\theta_{1}>r^{1 / 2}$. Note that, up to $t=t_{1}, \Omega_{t}\left(u_{n}^{M}\right)$ is star-shaped with respect to both $B_{r}\left(x_{0}\right)$ and $B_{r}\left(x_{1}\right)$. Hence $\Omega_{t}\left(u_{n}^{M}\right)$ contains the strip

$$
\Sigma:=\left\{x: x \in B_{r}(z), z=x_{0}+t e_{1}, t \in\left[0, r^{1 / 2}\right]\right\} .
$$

Let $\phi$ be the reflection with respect to the line parallel to $e_{2}$ and going through $x_{1}$. Then

$$
u_{n}^{M}\left(\phi(x), t_{1}\right) \leqslant u_{n}^{M}\left(x, t_{1}\right) \quad \text { in } D_{3}:=\left\{x:\left(x-x_{1}\right) \cdot e_{1} \leqslant 0\right\} .
$$

Hence, by Lemma B.1 we have

$$
u_{n}^{M}\left(\phi(x), t_{0}\right) \leqslant u_{n}^{M}\left(x, t_{0}\right) .
$$

If we combine (B.4) with the fact that $u_{n}^{M}\left(x, t_{0}\right)=0$ on the right hand side of $l_{0}$, then it follows that $\Omega_{t_{0}}\left(u_{n}^{M}\right)$ is contained in the channel of width at most $2 r^{1 / 2}$ and height $6 R$. Now the same argument as in Case 1 yields a contradiction if $r<(3 R)^{-6}$.

Lastly, suppose

$$
y_{1} \in B_{r}\left(x_{0}+\tau e_{1}\right) \cap \partial \Omega_{0} \quad \text { for some } \tau \in\left[0, r^{1 / 2}\right) .
$$


Let $l_{3}$ be the line parallel to $e_{2}$ containing $y_{1}$. Since $\Omega_{0}$ is convex and symmetric with respect to the $e_{1}$-axis, we have $u_{0}(x)=0$ on the right side of $l_{3}$. Therefore LemmaB.1 implies that for $t>0$,

$$
u_{n}^{M}(\cdot, t) \leqslant u_{n}^{M}(\phi(x), t) \quad \text { on the right side of } l_{3},
$$

where $\phi(x)$ is the reflection of $x$ with respect to $l_{3}$. Now one can proceed as in Case 2 to derive a contradiction.

REMARK One class of initial configurations covered by the above theorem are circular sectors

$$
\Omega\left(u_{0}\right)=\left\{r e^{i \theta}: 0 \leqslant r \leqslant R, 0 \leqslant \theta \leqslant \theta_{0}\right\}
$$

\section{Acknowledgments}

K.G. acknowledges support under NSF award DMS-0405596. I.K. acknowledges support under NSF award DMS-0627896.

\section{REFERENCES}

[C] Cox, R. G. The dynamics of the spreading of liquids on a solid surface. Part 1. Viscous flow. J. Fluid Mech. 168 (1986), 169-194. Zbl 0597.76102

[CL] Crandall, M. G., \& Lions, P.-L. Viscosity solutions of Hamilton-Jacobi equations. Trans. Amer. Math. Soc. 277 (1983), 1-42. Zbl 0599.35024 MR 0690039

[CJK] ChOI, S., JeRISON, D. S., \& KIM, I. C. Regularity for the one-phase Hele-Shaw problem from a Lipschitz initial surface. Amer. J. Math. 129 (2007), 527-582. Zbl pre05152576 MR 2306045

[G1] Glasner, K. B. A boundary integral formulation of quasi-steady fluid wetting. J. Comput. Phys. 207 (2005), 529-541. Zbl pre02183216 MR 2144627

[G2] Glasner, K. B. Homogenization of contact line dynamics. Interfaces Free Bound. 8 (2006), 523542. Zbl 1111.35134 MR 2283924

[Gr] GreEnspan, H. P. On the motion of a small viscous droplet that wets a surface. J. Fluid Mech. 84 (1978), 125-143. Zbl 0373.76040 MR 1206141

[Ho] Hocking, L. M., \& Miksis, M. J. Stability of a ridge of fluid. J. Fluid Mech. 247 (1993), 157-177. Zbl 0767.76019

[Hu] Hunter, J. K., Li, Z., \& ZhaO, H. Reactive autophobic spreading of drops. J. Comput. Phys. 183 (2002), 335-366. Zbl 1046.76036 MR 1947775

[K1] KIM, I. C. Uniqueness and existence results on the Hele-Shaw and Stefan problems. Arch. Ration. Mech. Anal. 168 (2003), 299-328. Zbl 1044.76019 MR 1994745

[K2] KIM, I. C. A free boundary problem with mean curvature, Comm. Partial Differential Equations 30 (2005). 121-138. Zbl 1069.35094 MR 2131048

[T] TANner, L. The spreading of silicone oil drops on horizontal surfaces. J. Phys. D 12 (1979), 14731484.

[Va] VÁzQuez, J. L. The free boundary problem for the heat equation with fixed gradient condition. Free Boundary Problems: Theory and Applications (Toledo, 1993), Pitman Res. Notes Math. Ser. 323, Longman, Harlow (1996), 277-302. Zbl 0867.35120 MR 1462990

[V] Volnov, O. V. Hydrodynamics of wetting. Fluid Dynamics 11 (1976), 714-721. 